May 2021

"On the evolution of male competitiveness"

Ingela Alger 


\title{
On the evolution of male competitiveness ${ }^{*}$
}

\author{
Ingela Alger ${ }^{\dagger}$
}

May 14, 2021

\begin{abstract}
Since a man's reproductive success depends on his ability to outcompete other men, male competitiveness may be expected to have been exposed to strong selective pressure throughout human history. Accordingly, the relatively low level of physical violence observed between men has been viewed as a puzzle. What could have limited the eagerness of men to out-compete each other? I study the evolution of male competitiveness in a model where men compete for both reproductive and productive resources. I show that high levels of male competitiveness are then consistent with evolution by natural selection if (a) the ecology is generous enough for men to supply little or no food to their children, (b) competing is not too costly in terms of productive resources, and (c) relatedness among males is low enough. While the main analysis takes women to passively accept the husband that emerges from the male-male competition, the results are qualitatively robust to allowing for female mate choice following the male-male competition game. Possible implications for our understanding of the evolution of marriage systems are discussed.
\end{abstract}

Keywords: male-male competition, competitiveness, evolution, monogamy, polygyny, parental care

JEL codes: D13, Z1, C73

${ }^{*}$ This paper is a heavily revised version of a paper circulated under the name "How many wives do men want? On the evolution of preferences over polygyny rates." I acknowledge funding from the European Research Council (ERC) under the European Union's Horizon 2020 research and innovation programme (grant agreement No 789111 - ERC EvolvingEconomics), as well as IAST funding from the French National Research Agency (ANR) under grant ANR-17-EURE-0010 (Investissements d'Avenir program). I am grateful to Donald Cox, Hillard Kaplan, Karen Kramer, and Michael Wilson for extensive comments and discussions. I also thank Alice Baniel, Alexis Chaine, Lena Edlund, Hanna Kokko, Jorge Peña, François Salanié, Jonathan Stieglitz, and audiences at EHBEA 2017, SEHO 2019, Max Planck Institute for Research on Collective Goods in Bonn, IAST, and Freie Universität Berlin for comments. Olivier Lision provided excellent research assistance.

${ }^{\dagger}$ Toulouse School of Economics, CNRS, University of Toulouse Capitole, and Institute for Advanced Study in Toulouse, France. ingela.alger@tse-fr.eu 


\section{Introduction}

In a species like Homo sapiens, where fertilization and gestation take place inside women's bodies, a man's reproductive success depends on the number of women he fertilizes, while a woman's reproductive success is limited by the number of children she can give birth to. This basic biological fact implies that males face a more severe competition for reproductive resources than women do. In fact, it is unclear what could limit the eagerness of males to out-compete each other, and the relatively low levels of physical violence observed between men have been viewed as a puzzle (Seabright, 2004). ${ }^{1}$ The theory proposed here sheds new light on this question. It centers on the premise that a full understanding of the evolution of male-male competition requires an understanding of how evolution by natural selection shapes male preferences over the number of wives ${ }^{2}$ in the first place, and how these preferences affect the men' willingness to compete against each other. Intuitively, one should expect lower levels of male-male competition if all men would prefer to have only one wife, than if they all preferred to have many wives. The goal of this paper is twofold: to endogenize men's preferences over polygyny rates, and to analyze the consequences of these preferences for the evolution of male competitiveness.

Following evolutionary logic, I posit that individuals seek to maximize reproductive success, defined as the expected number of children that survive to sexual maturity. For any given household composition, a man and his spouse(s) thus engage in parental care to this end. This is modeled as a non-cooperative game between the adults in the household. Equilibrium fertility and parental care choices in turn determine the number of wives a man would like to have if he could choose freely, a number which typically depends on exogenously given factors of the environment, or the ecology, in which the population evolves. I then use these results to examine the evolution of the willingness of men to compete against other men. Assuming that male degree of competitiveness is a trait transmitted from father to son, I adopt a standard evolutionary game theoretic framework (Weibull, 1995) to determine which degrees of competitiveness are compatible with evolution by natural selection. ${ }^{3}$ The strategies in the evolutionary game are the degrees of compet-

\footnotetext{
${ }^{1}$ I focus exclusively on competition between men at the individual level, not the group level, and hence I do not seek to explain the prevalence of wars. Moreover, the model is general enough to encompass several kinds of competition, ranging from physical combat to poetry contests. Hence, low levels of physical violence between men would not be the only empirical indicator of lack of competitiveness. Several studies have nonetheless shown that levels of physical violence between men tend to be higher in societies which permit polygyny than in societies which impose monogamy (see Henrich, Boyd, and Richerson, 2012, and the references therein).

2 The term "wife" is used for convenience only, since marriage has no function per se in the model. The key assumption is that both males and females engage in parental care, which is a reasonable assumption for humans and some other species (see Alger and Cox, 2013, for a review of the biology literature on parental care).

${ }^{3}$ While the mathematical model is silent as to whether the transmission is biological or cultural, recent evidence on the interacting effects of testosterone and cortisol levels on male willingness to compete (e.g., Knight et al., 2020) suggests that biological factors do matter (but since the mechanisms are not yet fully understood, father-son correlations are still unknown). Moreover, testosterone levels are on average lower in married than in unmarried men, and the lowest levels are found in married men with children (Alvergne, Faurie, and Raymond, 2009; Gettler et al., 2011). Theoretical analysis of transmission routes other than the purely vertical one considered here, which would be possible
} 
itiveness. Men are matched randomly into pairs to play, and each man's strategy in any matched pair determines whether he competes or refrains from competing. Men may differ in their degrees of competitiveness: in matched pairs with two non-competitive men, each man settles down and forms a monogamous household; by contrast, if at least one of the men in a matched pair is competitive the encounter ends with one winner, who takes over the women of the loser. I characterize the set of evolutionarily stable degrees of competitiveness. Several settings are considered. First, to reflect the fact that men's ability to acquire or destruct each other's productive resources has changed over the course of history, the stake of the competition may, besides women, include productive resources. Second, while I first adopt the standard evolutionary game theory framework in which matching between men is uniformly random, I also extend the model to capture the fact that humans live in groups between which there is limited migration. Furthermore, while the main analysis takes women to passively accept the husband that emerges from the male-male competition, I verify that the qualitiative nature of the results are robust to allowing for female mate choice following the male-male competition game, hence accounting also for the role that female preferences over household composition play.

Could evolution by natural selection ever be compatible with men refraining from unlimited competition for women? My model provides three novel answers to this question. First, and in stark contrast with the standard assumption that male reproductive success increases in the polygyny rate, I show that in some ecologies a man's reproductive success decreases with the number of women (although in other ecologies it is increasing or non-monotonic). This result is explained by men's investment of time and resources in their children and women's agency over their fertility. ${ }^{4}$ In ecologies where the man chooses to provision food, which is a fully rival good, men thus face a quantity-quality trade-off with respect to the number of wives. I show that the trade-off can be so severe that the man prefers to have one rather than several wives. ${ }^{5}$ This in turn has deep implications for the set of evolutionarily stable degrees of male competitiveness. If the prevailing strategy consists in being peaceful and if male reproductive success is higher with one than with two wives, it would not pay off to mutate towards a higher degree of competitiveness in order to acquire more wives. However, I further find that even in ecologies where male reproductive success decreases with the number of wives, the highest degree of competitiveness is typically also evolutionarily stable (more on this below, however). It is easy to see why: if the prevailing strategy consists in being maximally competitive, a man who mutates towards a lower degree of

if transmission is (fully or partly) cultural, is left for future research.

${ }^{4}$ While paternal provisioning is rare among mammals (Clutton-Brock, 1991), it did evolve at some point among our hominin ancestors, and I situate my model in the period of our evolutionary past that follows this ground-breaking development (see Gavrilets, 2012, and Alger et al., 2020, for theories of male provisioning in the hominin lineage). Regarding women's agency over fertility decisions, infanticide by men appears to have been applied mostly to nonrelated children (van Schaik and Janson, 2000).

${ }^{5}$ Clearly, this result hinges on the assumption that a man cannot impose different fertility rates on his wives, i.e., he cannot use some for reproduction and others for production. 
competitiveness is essentially guaranteed to live a mateless life.

Second, when the stake of the competition also includes productive resources, the nature of the competition matters. As intuition would suggest, the sustainability of lack of competitiveness is hampered by forms of competition whereby the winner's productive resources are bolstered (through the take-over of land for example), since this raises the reproductive success from having more than one wife. Likewise, the sustainability of lack of competitiveness is facilitated by forms of competition that entail a loss of productive resources (through injury for example), since this lowers the reproductive success from having more than one wife. However, even though lack of competitiveness may be evolutionarily stable, maximal competitiveness is also evolutionarily stable, for the same reason as above: if the prevailing strategy is maximal competitiveness, mutating towards a lower degree of competitiveness entails a mateless life.

Third, I show that the fact that our ancestors lived in groups of small size, which extended beyond the nuclear family and between which there was limited migration, should have favored non-competitive men. It is well known that a key implication of such population structurewhich is part of the environment of evolutionary adaptation of the human lineage (Van Schaik, 2016) - is that mutants, even when rare, face a higher probability of interacting with mutants than residents do, even absent any ability of individuals to choose the individuals with whom they interact. In the biology literature, this effect is usually quantified by the coefficient of relatedness (Wright, 1931), which measures the probability that interacting individuals share a common ancestor. ${ }^{6}$ I show that, although the highest degree of competitiveness is always evolutionarily stable when matching is uniformly random, it no longer is so when the coefficient of relatedness is high enough. The reason is that polygyny is always inefficient in the sense that overall average reproductive success is lower under polygyny than under monogamy. If the prevailing strategy consists in competing as much as possible, relatedness implies that rare mutants who compete less can reap the benefits of the reduced competition with a non-negligible probability, and thus outperform the more competitive men.

Brought together, the results of my model indicate that-absent inter-generational transmission of wealth and unbalanced sex ratios-male-male competition for wives (i.e., sexual partners into whose offspring the man invests resources) is consistent with evolution by natural selection only if (a) the ecology is generous enough for the male adult household member to provision little or no food to the offspring, (b) the competition does not reduce the winning male's productive resources too much, and (c) relatedness among males is low enough. Importantly, it is the combination of these three factors that is necessary for male-male competition for wives to be compatible with evolution by natural selection.

\footnotetext{
${ }^{6}$ See also Hamilton (1964), Frank (1998), and Rousset (2004). For economics models having analyzed the effects of such relatedness, see Bergstrom (1995, 2003), Alger and Weibull (2010, 2013), and Alger, Weibull, and Lehmann (2020).
} 
This paper contributes to two strands of literature. First, it contributes to the literature which hypothesizes that exogenously given factors in the "ecology" in which a population evolves can impact the selective pressure on behaviors. This quest, reminiscent of behavioral ecology (Tinbergen, 1963), and common in biology and evolutionary anthropology, has recently generated a large number of empirical studies in economics, which indicate that behavioral heterogeneity observed today may be traced to differential exposure to various factors in ancient times. Factors that have thus been examined empirically include production technologies (Alesina, Giuliano, and Nunn, 2013, BenYishay, Grosjean, and Vecci, 2017), sex ratios (Grosjean and Khattar, 2019), migration patterns (Spolaore and Wacziarg, 2009, Ashraf and Galor, 2013, Becker, Enke, Falk, 2020), and climatic risk (Durante and Buggle, 2020). The differences in several preference indicators across societies reported by Falk et al. (2018) are also consistent with the hypothesis. While none of these studies examines variation in male-male competition across societies, my model predicts that human societies may have diverged in this dimension a long time ago, the competition being more fierce in some societies than in others. It also provides a rationale for such a divergence. More generally, this paper joins the theoretical literature that investigates how evolutionary forces may have shaped human preferences (Frank, 1987, Güth and Yaari, 1992, Bergstrom, 1996, Robson, 2001, 2002, Dekel, Ely, and Yilankaya, 2007, Heifetz, Shannon, and Spiegel, 2007, Rayo and Becker, 2007, and $\mathrm{Wu}, 2020)$. In particular, it expands the set of preferences that have been examined, previous studies having analyzed the impact of shock distributions on the discrepancy between decision and experienced utility (Robson and Samuelson, 2011); of the harshness of the environment on intra-family altruism (Alger and Weibull, 2010); of the advent of agriculture on the willingness to defend private property (Bowles and Choi, 2019); of migratory patterns on preferences governing behavior in social interactions (Alger, Weibull, and Lehmann, 2020).

Second, my findings complement those in the existing literature on the historical evolution of polygyny rates, which examines factors that I do not consider, such as returns to wealth (Bergstrom, 1994a), the dilution of land ownership due to polygyny (Lagerlöf, 2005), male heterogeneity in income (Grossbard, 1976, Bergstrom, 1994b) population growth (Tertilt, 2005, 2006), sex ratios (Edlund and Ku, 2013), heterogeneity in women's human capital (Grossbard, 1980, Gould, Moav, and Simhon, 2008), social unrest (Lagerlöf, 2010, De La Croix and Mariani, 2015), and free-riding in paternal care (Francesconi, Ghiglino, and Perry, 2016). The closest model to mine is that by Ross et al. (2018), which also features men who provide both a rival and a non-rival good to their offspring, and where the relative importance of these goods is linked to the ecology in which the population evolves. Importantly, however, their model (as well as the models cited above) has exogenously given male preferences over polygyny rates and all disregard men's willingness to compete for wives as a fundamental driver of male heterogeneity. Moreover, they all assume that men would always want more wives, should their price be nil. By contrast, the model proposed here derives male preferences over polygyny rates from first principles and analyzes men's 
willingness to compete for wives as an inherited trait subject to natural selection. The central finding is that male reproductive success can decrease with the polygyny rate. This model thus provides a novel explanation for why a complete lack of male-male competition and the associated monogamy can be consistent with evolution by natural selection.

The model is presented and analyzed in the next two sections, which are followed by a discussion of the implications of the results for our understanding of the evolution of marriage systems, and some concluding remarks. All the mathematical proofs can be found in the Appendix.

\section{The child production stage}

I model a population in which each individual lives for at most two periods; as a non-productive and non-reproductive child in the first period, and-conditional on surviving to sexual maturityas a productive and reproductive adult in the second period. In each generation the sex ratio is assumed to be balanced at birth, ${ }^{7}$ and for simplicity also at the beginning of the adult period. The analysis focuses on behaviors in the adult period, which has two stages: a male-male competition stage followed by a child production stage. To simplify the analysis I first consider a model in which women passively accept to settle down in the household that the outcome of the male-male competition dictates, but I then lift this assumption.

In the male-male competition stage, men compete for women, and possibly also for productive resources. The competition sorts men into two categories: those with and those without mates, where those with mates all have the same number of mates. At the beginning of the child production stage, there is thus a number of households, each composed of one man and his (identical) spouse(s). Within each such household the man and his spouse(s) then engage in tasks, the goal of each individual being to maximize reproductive success, defined as the expected number of offspring that survive to sexual maturity. ${ }^{8}$

While several alternative male-male competition scenarios will be analyzed, the child production stage is the same for all of them. Since the reproductive success a man obtains for any given household composition impacts the benefit of engaging in competitive behavior in the male-male competition stage, I proceed by backward induction and first analyze the child production stage. This will allow me to characterize how male reproductive success varies with the number of wives.

\footnotetext{
${ }^{7}$ Although the sex ratio can vary significantly from year to year in small populations (see, e.g., Kramer, Schacht, and Bell, 2017), an (almost) balanced sex ratio at birth is empirically verified when measured for large populations where sex-specific abortions are not conducted. A balanced sex ratio is also predicted by evolutionary theory (Fisher, 1930).

${ }^{8}$ In reality mating success of offspring who have survived to sexual maturity also matters for an adult's reproductive success. I disregard this here, by letting mate matching be random rather than based on choice.
} 


\subsection{The household game}

Consider a household with one man and his $k \geq 1$ spouse(s) entering the child production stage. ${ }^{9}$ In the main analysis a woman has no say when it comes to the number of wives in the household. ${ }^{10}$ However, I assume that she has full agency over her fertility and childcare decisions (alternative assumptions will be discussed below). Each woman and each man is endowed with some resources that they devote to parental investment. While in reality these resources may be multi-dimensional, for simplicity I assume that they can be aggregated into one dimension, and I will refer to this resource as time. Let the time budgets available for parental investment be $X \in(0,1]$ for each woman and $Y \in(0,1]$ for the man. I assume that there is sexual division of labor: the man specializes in protection, women specialize in care, and both the man and the women provision the children with food (although not necessarily the same kind of food). ${ }^{11}$ Letting $G_{j}$ and $C_{j}$ denote the amounts of food and care that child $j$ receives from its mother, and $H_{j}$ and $P_{j}$ the amounts of food and protection received from its father, I assume that the probability that the child (whether a boy or a girl) survives to sexual maturity is ${ }^{12}$

$$
\max \left\{0,1-b n_{j}\right\} \cdot S\left(C_{j}, G_{j}, H_{j}, P_{j}\right)
$$

where $n_{j}$ is the total number of children that $j$ 's mother gave birth to, and

$$
S\left(C_{j}, G_{j}, H_{j}, P_{j}\right)=P_{j}^{\lambda} \cdot C_{j}^{\sigma} \cdot\left(\rho G_{j}+H_{j}\right)^{\tau},
$$

where $\lambda, \sigma, \tau \in(0,1) .{ }^{13}$ The first term in (1) reflects the physical toll of giving birth, where $1 / b$ can be thought of as the maximum number of children a woman can have before she dies with certainty (in which case the children die since the mother's inputs are essential). ${ }^{14}$ The function in (2) describes how the parental inputs affect the survival probability. It captures two realistic

\footnotetext{
${ }^{9}$ For simplicity, interactions between households, divorce, and unfaithfulness are ruled out by assumption. It would clearly be desirable to endogenize the degree of unfaithfulness, but this has to be left for future research. For the time being, one interpretation of the benchmark model considered here is that there is strong social control (for instance, although they are not explicitly modeled here, there may be grand-parents who monitor how their children behave in the adult stage, or the females monitor each other). Moreover, most estimates of current extra-pair paternity rates are low, ranging between 0 and $11 \%$ across societies (see Simmons et al., 2004, and Anderson, 2006).

${ }^{10}$ The consequences of female choice will be examined in Subsection 3.4.

${ }^{11}$ The terms protection and care should be interpreted broadly. Thus, protection may include shelter construction and maintenance, active protection against predators, as well as the transmission of human capital pertaining to such activities. Likewise, care may include the production and mending of clothes, storytelling, as well as the transmission of knowledge about social rules, plants, and animals.

${ }^{12}$ Together with the assumption that the sex ratio is balanced, this assumption implies that the expected average reproductive success of a newborn girl equals that of a newborn boy, even though polygyny will imply that some boys will obtain a higher reproductive success than other boys.

${ }^{13}$ Admittedly, more general setups could be imagined. However, this model specification is sufficient to obtain the novel insights that this paper contributes to the literature.

${ }^{14}$ Here the physical toll is modeled as a scaling factor: ceteris paribus, the more children a woman has, the smaller is the survival probability of each of her children. Other specifications could be conceivable, including ones where the physical toll would have an impact on female productivity. Such alternative specifications are left for future research.
} 
features. First, food, protection, and care are all essential goods: food is useless unless some protection and some care is provided, and vice versa. Second, the food provided by the mother and the father are perfect substitutes, although the food provided by the mother relative to that provided by the father may be more important $(\rho>1)$, equally important $(\rho=1)$, or less important $(\rho<1)$; this may depend on the nutritional attributes of different kinds of food in the location occupied by the population at hand. The parameters $\lambda, \sigma$, and $\tau$ measure how protection, care, and total food intake, respectively, impact the survival probability. These parameter values would typically also depend on the local environment: protection and care is relatively more important if there are many predators around or if there are many dangers that children need to learn to avoid.

The amounts of food, care, and protection that a child $j$ receives, as captured by the vector $\left(C_{j}, G_{j}, H_{j}, P_{j}\right)$ (see (1) and (2)), depend on the production decisions of his or her parents, to which I now turn. Denote by $y \in[0, Y]$ the time that the man devotes to producing food, so that $Y-y$ is spent on protecting the household. Likewise, denote by $x_{i}$ the time that wife $i=1, \ldots, k$ devotes to producing food, and $X-\phi x_{i}$ the time that she spends on caring, where the parameter $\phi \in(0,1)$ measures the extent to which a woman may produce food while providing care (historically women have tended to engage in gathering plant-based food, which does not run away from crying babies, thus rendering food production compatible with simultaneous child care). Letting $n_{i}$ denote wife $i$ 's number of offspring, the household's child production allocation is summarized by the vector $(\boldsymbol{n}, \boldsymbol{x}, y)$, where $\boldsymbol{n}=\left(n_{1}, \ldots, n_{k}\right)$ and $\boldsymbol{x}=\left(x_{1}, \ldots, x_{k}\right)$. If all the women adopt the same fertility and the same time allocation, i.e., if $n_{i}=n$ and $x_{i}=x$ for all $i \in\{1,2, \ldots, k\}$, the child production allocation will be called female-symmetric and be denoted $(n, x, y)$.

Assuming that each adult divides the goods he or she produces equally among his or her children, I use an index $i$ to refer to each of the $n_{i}$ children of wife $i=1, \ldots, k$. Child $i$ receives the amount

$$
H_{i}=\frac{\theta y}{\sum_{\ell=1}^{k} n_{\ell}}
$$

of food from its father, where $\theta>0$ is the marginal return to male effort devoted to producing food, and the amount

$$
G_{i}=\frac{\gamma x_{i}}{n_{i}}
$$

of food produced by its mother, where $\gamma>0$ is the marginal return to female effort devoted to gathering. ${ }^{15}$ In other words, the food brought home by the father is divided equally across all his children, while the food brought home by each mother is divided only across her own children. I

\footnotetext{
${ }^{15}$ Constant returns to effort are perfectly compatible with specialization, if, for instance, the marginal return to male effort devoted to gathering and to caring is strictly lower than that of a female, and the marginal return to female effort devoted to hunting and to protecting is strictly lower than that of the male. However, the assumption may be inappropriate for pastoralist societies, where there may be increasing returns to men's efforts on herding.
} 
assume that $\rho \gamma+\theta \leq 1$; this ensures that $S$ always takes a value between 0 and 1 .

While food is a rival good, care and protection may be non-rival; for instance, a wall around the village protects all the children equally well, and a lesson about poisonous plants may benefit several children simultaneously. Formally, let

$$
P_{i}=\frac{Y-y}{\left(\sum_{\ell=1}^{k} n_{\ell}\right)^{\alpha}}
$$

be the amount of protection that each child of wife $i$ receives, where $\alpha \in[0,1]$ is the degree of rivalry of protection. In the extreme case where $\alpha=0$, protection is fully non-rival, and each child receives the full benefit of the total amount of protection produced by the father: $p(y, Y, \boldsymbol{n})=Y-y$. At the other extreme, if $\alpha=1$, protection is a fully rival good, and each child receives an equal share of the total amount produced: $p(y, Y, \boldsymbol{n})=(Y-y) /\left(\sum_{j=1}^{k} n_{j}\right)$. Likewise, the amount of care that a child of wife $i$ receives is

$$
C_{i}=\frac{X-\phi x_{i}}{n_{i}^{\beta}}
$$

where $\beta \in[0,1]$ measures the degree of rivalry of care. If $\beta=0$, care is fully non-rival, while if $\beta=1$, it is a fully rival good.

I assume that all the adult members of the household make independent decisions, and that all seek to maximize own reproductive success. Thus, each woman $i=1, \ldots, k$ chooses her fertility $n_{i}$ and her time allocation $x_{i}$, taking the man's and the other women's time allocations as given, and the man chooses his time allocation $y$, taking the women's fertility and time allocation decisions as given. Formally, given $k$, the situation at hand is thus a simultaneous-move game with $k+1$ players; each female player $i=1, \ldots, k$ chooses a strategy $\left(n_{i}, x_{i}\right) \in[0,1 / b] \times[0, X]$, while the male player chooses a strategy $y \in[0, Y]$. The specification of each individual's reproductive success, which is his or her payoff in the game, as a function of the strategy profile $(\boldsymbol{n}, \boldsymbol{x}, y)$, completes the description of the game. The reproductive success of wife $i$ writes

$$
F\left(n_{i}, x_{i}, \boldsymbol{n}_{-i}, y\right)=n_{i} \cdot \max \left\{0,1-b n_{i}\right\} \cdot s\left(n_{i}, x_{i}, \boldsymbol{n}_{-i}, y\right),
$$

where, from (2)-(6),

$$
s\left(n_{i}, x_{i}, \boldsymbol{n}_{-i}, y\right)=\left[\frac{Y-y}{\left(\sum_{j=1}^{k} n_{j}\right)^{\alpha}}\right]^{\lambda} \cdot\left(\frac{X-\phi x_{i}}{n_{i}^{\beta}}\right)^{\sigma} \cdot\left[\frac{\rho \gamma x_{i}}{n_{i}}+\frac{\theta y}{\left(\sum_{j=1}^{k} n_{j}\right)}\right]^{\tau},
$$

while the reproductive success of the man is the sum of his wives' reproductive successes:

$$
M(\boldsymbol{n}, \boldsymbol{x}, y)=\sum_{j=1}^{k} F\left(n_{j}, x_{j}, \boldsymbol{n}_{-j}, y\right)=\sum_{j=1}^{k} n_{j} \cdot \max \left\{0,1-b n_{j}\right\} \cdot s\left(n_{j}, x_{j}, \boldsymbol{n}_{-j}, y\right) .
$$


Let $\Gamma$ denote the household game thus described. ${ }^{16}$

This completes the description of the decisions taken within a household and their consequences. In the extremely long run, everything in life, including the features and the prevalence of animals and plants eaten by humans, is endogenous. However, the speeds at which different elements of a human society evolve, differ. In the model, I assume that the production technology, the degrees of rivalry of protection and care, the child survival probability function, the amount of female labor resources, and the physical toll due to childbirth are exogenous and fixed, and I refer to the associated set of parameters as the ecology. Formally, then, the ecology is the vector $\omega=(b, \phi, \sigma, \lambda, \tau, X, \alpha, \beta, \gamma, \theta, \rho)$. The ecology determines how parental time allocations and the fertility rate together determine male reproductive success, and below it will be seen how this in turn affects the set of sustainable polygyny rates. For further use below, let the set of ecologies be denoted $\Omega$, i.e., $\Omega=\left\{\omega \in(0,1)^{2} \times(0,1)^{3} \times(0,1] \times[0,1]^{2} \times \mathbb{R}^{3} \quad \mid \quad \rho \gamma+\theta \leq 1\right\} .{ }^{17}$

\subsection{Equilibria of the household game}

Assuming the game is one of complete information, the following result obtains:

Proposition 1. For any ecology $\omega$, any number of wives $k \geq 1$, and any amounts of the male resources $Y$, there exists a unique Nash equilibrium of the household game $\Gamma$. Moreover, this equilibrium is femalesymmetric.

In words, in any household there is a unique Nash equilibrium strategy profile, and, moreover, at this equilibrium strategy profile all the women choose the same number of children and the same time allocation. While the model does not allow to obtain a closed-form solution for the equilibrium number of children, $n^{*}$, the expressions for the equilibrium time allocations, $x^{*}$ and $y^{*}$, are as follows $\left(x^{*}\right.$ and $y^{*}$ are stated as functions of the number of wives $k$ and the amount of male resources $Y$, for these are the two variables that will be determined endogenously by the malemale competition). To simplify the notation let $\mu \equiv \theta /(\rho \gamma)$; this ratio measures the importance of food contributed by the man relative to that contributed by the women. Two cases arise: if $\frac{\tau}{\sigma+\tau} \leq \phi$,

$$
\left(x^{*}(k, Y), y^{*}(k, Y)\right)=\left\{\begin{array}{l}
\left(\frac{X \tau}{\phi(\sigma+\tau)}, 0\right) \text { if } k \geq \frac{\mu \phi(\sigma+\tau)}{\lambda} \cdot \frac{Y}{X} \\
\left(\frac{X(\lambda+\tau) k-Y \sigma \phi \mu}{k \phi(\lambda+\sigma+\tau)}, \frac{Y \mu \phi(\sigma+\tau)-X \lambda k}{\mu \phi(\lambda+\sigma+\tau)}\right) \text { if } k \in\left[\frac{\mu \phi \sigma}{\lambda+\tau} \cdot \frac{Y}{X}, \frac{\mu \phi(\sigma+\tau)}{\lambda} \cdot \frac{Y}{X}\right] \\
\left(0, \frac{Y \tau}{\lambda+\tau}\right) \text { if } k \leq \frac{\mu \phi \sigma}{\lambda+\tau} \cdot \frac{Y}{X}
\end{array}\right.
$$

\footnotetext{
${ }^{16}$ For any given polygyny rate $k$ and female-symmetric fertility $n$, the goals of the man and his spouse(s) are aligned; hence, the time allocation could have been modeled as resulting from a cooperative game instead of a non-cooperative one without affecting the results. Relatedly, in an earlier version of the model, I let the man impose his preferred fertility on his spouse(s); the qualitative nature of the results were the same as those reported here.

${ }^{17}$ For some parameter constellations the reproductive success is so low that to obtain sensible numerical examples the expression in (7) must be multiplied by some number above 1 . This does not affect the results in any way, however, since the trade-offs are unaffected by such a positive factor.
} 
while if $\frac{\tau}{\sigma+\tau}>\phi$,

$$
\left(x^{*}(k, Y), y^{*}(k, Y)\right)=\left\{\begin{array}{l}
(X, 0) \text { if } k \geq \frac{\mu \tau}{\lambda} \cdot \frac{Y}{X} \\
\left(X, \frac{Y \tau}{\lambda+\tau}-\frac{X k \lambda}{\mu(\lambda+\tau)}\right) \text { if } k \in\left[\frac{\mu \sigma \phi}{\lambda+\tau-\phi(\lambda+\sigma+\tau)} \cdot \frac{Y}{X}, \frac{\mu \tau}{\lambda} \cdot \frac{Y}{X}\right] \\
\left(\frac{X(\lambda+\tau) k-Y \sigma \phi \mu}{k \phi(\lambda+\sigma+\tau)}, \frac{Y \mu \phi(\sigma+\tau)-X \lambda k}{\mu \phi(\lambda+\sigma+\tau)}\right) \text { if } k \in\left[\frac{\mu \sigma \phi}{\lambda+\tau} \cdot \frac{Y}{X}, \frac{\mu \sigma \phi}{\lambda+\tau-\phi(\lambda+\sigma+\tau)} \cdot \frac{Y}{X}\right] \\
\left(0, \frac{Y \tau}{\lambda+\tau}\right) \text { if } k \leq \frac{\mu \sigma \phi}{\lambda+\tau} \cdot \frac{Y}{X} .
\end{array}\right.
$$

Inspection of these expressions immediately reveals the following property, which will turn out to play an important role in the subsequent analysis:

Corollary 1. For any ecology $\omega$, any number of wives $k \geq 1$, and any amounts of the male resources $Y$, $\frac{\partial y^{*}(k, Y)}{\partial k} \leq 0$.

In words, the amount of resources that the man spends on food production, $y^{*}(k, Y)$, is (weakly) decreasing in $k$. This property arises because an increase in the number of wives $k$ implies that the man's food output is shared between a larger number of children. It is thus as if the man's ability to produce food relative to that of women was reduced. As a result, an increase in $k$ reduces the man's incentive to engage in food production. For $k$ large enough, all the food is produced by the women $\left(y^{*}(k, Y)=0\right)$. As will be seen below this feature is intimately linked with the qualitative characteristics of male preferences over polygyny rates.

The other comparative statics—which follow from simple calculations that are omitted-are also intuitive. A higher marginal benefit from producing food $(\tau)$ induces both the man and his spouse(s) to spend more time on food production. Likewise, the man spends less time and the women more time on food production in ecologies where protection is more important (a higher $\lambda$ ), while the opposite occurs in ecologies where female care is more important (a higher $\sigma)$. Similarly, in ecologies with greater economies of scope between the two female activities (a lower $\phi$ ), the cost for women of allocating time away from caring is smaller, and hence, female food production is larger; this in turn entails a smaller marginal effect of male food production on child success, and hence the man devotes less time to food production. Finally, men engage more in food production relative to women, the more efficient food providers they are compared to women, i.e., the larger is $\mu=\theta /(\rho \gamma)$. These results are collected in the following proposition.

Proposition 2. Ceteris paribus, $x^{*}$ is non-decreasing in $\tau$ and $\lambda$, and non-increasing in $\phi$, $\sigma$, and $\mu$, while $y^{*}$ is non-decreasing in $\tau, \sigma, \phi$, and $\mu$, and non-increasing in $\lambda$. Neither $x^{*}$ nor $y^{*}$ depend on $\alpha, \beta$, or $n$.

In order to prepare the ground for the analysis of the male-male competition stage below, I now ask the following question: if a man could freely choose the number of wives $k$, how many would he choose? The seemingly obvious answer is that the man must always benefit from an increase in the number of wives. However, this turns out not to be true in general. 
The equilibrium child production allocation being unique (see Proposition 1) the man's reproductive success is uniquely determined for any number of wives $k$ and any amount of male labor resources $Y$. Let $M^{*}:[0,+\infty) \times(0,1]$ denote the mapping that to each $(k, Y)$ associates the equilibrium male reproductive success, i.e.,

$$
M^{*}(k, Y)=M\left(\boldsymbol{n}^{*}(k, Y), \boldsymbol{x}^{*}(k, Y), y^{*}(k, Y)\right),
$$

and note that $M^{*}(k, Y)>0$ for any $k>0$. The following proposition describes how $M^{*}$ varies with $k$, for a given amount of male labor resources $Y$.

Proposition 3. For any $Y \in(0,1]$ there exists a partition $\left\{\Omega_{1}, \Omega_{2}, \Omega_{3}\right\}$ of $\Omega$ such that $\Omega_{1}, \Omega_{2}$, and $\Omega_{3}$ are all non-empty, and:

(1) for any $\omega \in \Omega_{1}, M^{*}(k, Y)$ is non-decreasing in $k$;

(2) for any $\omega \in \Omega_{2}$, there exists $k^{*}(\omega)>1$ such that $M^{*}(k, Y)$ is strictly decreasing in $k$ for all $k<k^{*}(\omega)$;

(3) for any $\omega \in \Omega_{3}, M^{*}(k, Y)$ is strictly decreasing in $k$.

Men face a quantity-quality trade-off, because producing more children (by having more wives) entails a decrease in the survival probability of children. The proposition says that: (A) the tradeoff can be so severe that male reproductive success declines if he adds more wives, and (B) the trade-off is most severe for low polygyny rates. Specifically, depending on the ecology, male reproductive success is either (1) monotonically increasing; (2) decreasing for low or all polygyny rates. For ecologies in $\Omega_{2}$, while in all numerical examples I have explored male reproductive success is increasing for values of $k$ above $k^{*}(\omega)$, I have not been able to prove this generally. This is due to the lack a closed-form solution for the equilibrium fertility rate $n^{*}$, which also explains why a characterization of the partition $\left\{\Omega_{1}, \Omega_{2}, \Omega_{3}\right\}$ of $\Omega$ referred to in Proposition 3 cannot be obtained. As a result it proves useful to study a modified household game, in which the man imposes both his preferred time allocation and his preferred (common) fertility rate on his spouse(s). The following lemma, which reports the results of this analysis, refers to this threshold value for the degree of rivalry of male protection (all the arguments necessary to prove this lemma are found in the proof of Proposition 3):

$$
\hat{\alpha} \equiv \frac{\rho \gamma x^{*}(1, Y)+(1-\tau) \theta y^{*}(1, Y)}{\lambda\left[\rho \gamma x^{*}(1, Y)+\theta y^{*}(1, Y)\right]} .
$$

Lemma 1. Consider a modified household game, in which the man imposes both his preferred time allocation and his preferred (common) fertility rate on his spouse(s). Then, for any $(k, Y)$, he would choose $\left(x^{*}(k, Y), y^{*}(k, Y)\right)$, i.e., the Nash equilibrium time allocation of household game $\Gamma$, and he would choose at least as many children per wife as that chosen in game $\Gamma$. Letting $\widetilde{M}(k, Y)$ denote the reproductive success that the man would achieve in this modified game:

(i) if $y^{*}(1, Y)=0$ or if $\alpha \leq \hat{\alpha}$, then $\frac{\partial \widetilde{M}(k, Y)}{\partial k} \geq 0$ for all $k \geq 1$ (the inequality being strict if $y^{*}(1, k)=0$ 
and $\alpha \lambda \neq 1$, or if $\alpha<\hat{\alpha})$;

(ii) if $y^{*}(1, k)>0$ and $\alpha>\hat{\alpha}$, then there exists $\hat{k}>1$ such that $\frac{\partial \widetilde{M}(k, Y)}{\partial k}<0$ for all $k \in(1, \hat{k})$ and $\frac{\partial \widetilde{M}(k, Y)}{\partial k}>0$ for all $k>\hat{k}$;

(iii) if $\alpha \lambda=1$, then $\widetilde{M}(1, Y) \geq \widetilde{M}(k, Y)$ for all $k>1$, the inequality being strict if and only if $y^{*}(1, Y)>0$.

Since $\widetilde{M}$ provides an upper bound for the equilibrium male reproductive success $M^{*}$ in game $\Gamma$, this lemma generates the following insights.

First, it shows that a key variable behind the result that male reproductive success is decreasing in the polygyny rate for some parameter values (see Proposition 3) is the man's involvement in food production. To see this, note that in Lemma $1 y^{*}(1, k)>0$ is a necessary condition for male reproductive success to be strictly decreasing in the polygyny rate for some parameter values. The intuition is as follows. Any time spent by the man on food production means that he uses resources to produce a fully rival good. This in turn renders the quantity-quality trade-off more severe than if he spent all his resources on producing the somewhat non-rival good protection. Since, as discussed above (see Proposition 1), male food production declines with the number of wives, this severe trade-off can only appear for low polygyny rates. As the number of wives grows large enough, the man stops engaging in food production, and adding more wives must then be beneficial (unless protection is almost fully rival). This explains why, for given male labor resources $Y$, male reproductive success cannot be increasing for low and decreasing for high polygyny rates.

Second, Lemma 1 reveals that the degree of rivalry of protection, $\alpha$, also plays a central role. The less rival is protection, the more the man stands to benefit from an increase in the number of wives. In the extreme case where protection is fully non-rival $(\alpha=0)$, he benefits from an increase in $k$ at all polygyny levels. By continuity, the same result obtains as long as $\alpha$ is sufficiently small. Thus, $\alpha$ must be sufficiently large for male reproductive success to be decreasing for some polygyny rates.

Third, Lemma 1 shows that the existence of ecologies where equilibrium male reproductive success decreases in the polygyny rate reported in Proposition 3 does not hinge on the assumption that women have agency over fertility and time allocation decisions, since it obtains even when the man can impose his preferred (common) fertility rate and time allocation on the women. ${ }^{18}$

I conclude the analysis of the child production stage by stating an additional set of results for the original household game $\Gamma$ in the following proposition (whose trivial proof is omitted):

\footnotetext{
${ }^{18}$ Clearly, however, the qualitative nature of the results reported in Proposition 3 would be jeopardized if a man could impose zero fertility on some wives and use these as labor resources to raise the children he sires with the other wives. The female agency assumption is justified for several reasons, however. First, a woman who is told to have no children has extremely strong incentives to flee. Second, even if such a woman stays with her husband, she has no incentive to perform the production of food and care diligently. Third, absent efficient contraceptive methods, the man would have to refrain from consummating the marriage with these wives, and it is not clear how realistic such an assumption would be. Of course, one could counter-argue that the man can resort to consummation with all wives cum discriminatory infanticide; however, this would likely simply worsen the two preceding issues.
} 
Proposition 4. For any given ecology $\omega \in \Omega$ :

(1) holding $Y$ fixed, $M^{*}(2 k, Y) \leq 2 M^{*}(k, Y)$, and the inequality holds as an equality if and only if male protection is fully non-rival $(\alpha=0)$ and the man devotes no time to food production $\left(y^{*}(k, Y)=0\right.$ for any $k \geq 1$ );

(2) holding $k \geq 1$ fixed, $M^{*}(k, Y)$ is continuous and strictly increasing in male labor resources $Y$, and $\lim _{Y \rightarrow 0} M^{*}(k, Y)=0$;

(3) holding $Y$ fixed, $\partial F^{*}(k, Y) / \partial k \leq 0$, and the inequality is strict unless male protection is fully nonrival $(\alpha=0)$ and the man devotes no time to food production $\left(y^{*}(k, Y)=0\right.$ for any $\left.k \geq 1\right)$.

The proposition says first that returns to wives are decreasing except in the extreme case where protection is fully non-rival and the man devotes all his time to providing protection, in which case returns are constant. The second result establishes that male reproductive success is increasing in the productive resources he controls. Finally, a woman's reproductive success is strictly decreasing in the polygyny rate in her household, except in the extreme case where protection is fully non-rival and the man devotes all his time to providing protection.

I am now in a position to analyze the first stage of the adult period, namely, the male-male competition stage.

\section{The male-male competition stage}

In the male-male competition stage men compete for access to women. While competition can also affect productive resources, I first analyze the case where it does not. Throughout, all the women are taken to be identical. Men are also identical when entering the competition stage, except potentially in their eagerness to compete, which is the trait whose evolution I analyze. Women are assumed to simply accept to join the household to which the male-male competition gives rise; the consequences of lifting this assumption will be examined in Subsection 3.4.

\subsection{Competition when only women are at stake}

To model male-male competition I posit an evolutionary game-the competition game-which concerns the male part of the population. Adopting a standard evolutionary game theoretic approach (e.g., Weibull, 1995), I assume that there is a continuum of male individuals and that each man is "programmed" to play a certain strategy, which may be interpreted as his eagerness to compete, or degree of competitiveness, inherited from his father. ${ }^{19}$ The competition game sorts men into two categories: "winners" and "losers." The winners get an equal number of wives each, while the losers remain mateless (or die, depending on the nature of the competition). Hence, this game

\footnotetext{
${ }^{19}$ In a more general model, each man could be equipped with preferences guiding his behavior in the competition game, and the transmitted trait would be the preferences. The simpler approach adopted here is in some settings equivalent to such preference evolution, and it also provides a useful benchmark (Alger and Weibull, 2013, 2019).
} 
endogenizes the allocation of women across men. The objective of the analysis is to determine the set of evolutionarily stable degrees of competitiveness. The nature of the competition-i.e., whether it takes the form of physical combat or a poetry contest, for example-is exogenous to the model.

To capture the main forces present in this model, it is sufficient to consider the simplest possible competition game, with only two pure strategies. Specifically, suppose that when entering the adult stage (from the teenage years) each man has one girlfriend, and that men are then (uniformly) randomly matched into pairs to play a simultaneous-move game with two pure strategies, Compete and Surrender. The strategy profile used in a matched pair determines the number of women accruing to each man. Thus, if both men play Surrender each gets to marry his teenage sweetheart. If at least one man plays Compete, then one of them gets to marry both girlfriends while the other one becomes mateless and remains so forever. A man who plays Compete wins with probability 1 if the other plays Surrender, and with probability $1 / 2$ if the other plays Compete.

In a matched pair where the strategy profile (Surrender, Surrender) is used, two monogamous households are thus formed. In the child production stage this gives rise to expected male reproductive success $M^{*}(1, Y)$ for each of these men. Likewise, in a matched pair where at least one player plays Compete, one bigynous household is formed, and the lucky man obtains expected male reproductive success $M^{*}(2, Y)$ while the unlucky man gets zero reproductive success. Accordingly, the payoffs in the evolutionary game are those in the matrix in Figure 1 (since this is a symmetric game, only the row player's payoffs are shown).

\begin{tabular}{|c|c|c|}
\hline & Surrender & Compete \\
\hline Surrender & $M^{*}(1, Y)$ & 0 \\
\hline Compete & $M^{*}(2, Y)$ & $\frac{1}{2} M^{*}(2, Y)$ \\
\hline
\end{tabular}

Figure 1. Payoffs in the competition game with constant productive resources

Let $r \in\{0,1\}$ denote a (pure) strategy in the evolutionary game, where $r=0$ means Surrender and $r=1$, Compete. Allow for mixed strategies and write $\zeta \in[0,1]$ for the probability of playing Compete. Now ponder the following thought experiment: suppose that a given strategy $\zeta$, the "resident" strategy, is used by almost everyone in the population, except for a small share $\varepsilon>0$ of individuals who use another strategy $\zeta^{\prime}$, the "mutant" strategy. Is there any resident strategy $\zeta$ that outperforms every possible "mutant" strategy $\zeta^{\prime} \in[0,1], \zeta^{\prime} \neq \zeta$, in the sense that those who carry the resident strategy get a strictly higher reproductive success on average than those who carry the mutant strategy, when mutants are rare? In other words, are there any evolutionarily stable strategies (ESS) in this evolutionary game? As an illustration, suppose that Compete is the resident strategy, present in a share $1-\varepsilon$ of the population, and Surrender the mutant one, present in a share $\varepsilon$ of the population. Compete is evolutionarily stable against Surrender if there 
exists some threshold value $\bar{\varepsilon}>0$ such that for all $\varepsilon \in(0, \bar{\varepsilon})$, the average reproductive success of a resident strictly exceeds that of a mutant: ${ }^{20}$

$$
(1-\varepsilon) \cdot \frac{M^{*}(2, Y)}{2}+\varepsilon \cdot M^{*}(2, Y)>\varepsilon \cdot M^{*}(1, Y)
$$

Likewise, Surrender is evolutionarily stable against Compete if for all $\varepsilon$ close enough to zero:

$$
(1-\varepsilon) \cdot M^{*}(1, Y)>(1-\varepsilon) \cdot M^{*}(2, Y)+\varepsilon \cdot \frac{M^{*}(2, Y)}{2} .
$$

The following proposition fully characterizes the set of evolutionarily stable strategies.

Proposition 5. In the competition game with constant productive resources:

(i) $\zeta=0$ is evolutionarily stable if and only if the ecology $\omega$ is such that:

$$
M^{*}(1, Y)>M^{*}(2, Y) \text {. }
$$

(ii) No mixed strategy $\zeta \in(0,1)$ is evolutionarily stable in any ecology $\omega \in \Omega$.

(iii) $\zeta=1$ is evolutionarily stable in any ecology $\omega \in \Omega$.

In other words, in some circumstances a population consisting of non-competitive men can resist the invasion of competitive men. Under constant productive resources, the ecology in which the population evolves is shown to be central: Surrender is evolutionarily stable in ecologies where men achieve a higher reproductive success with one than with two wives, given constant productive resources $Y$ (condition (16)). To see why, suppose that Surrender is the resident strategy. Any mutant-who plays Compete - then almost surely achieves reproductive success $M^{*}(2, Y)$, because he is almost surely matched with a resident, who Surrenders. However, the vast majority of residents are matched with other residents, and they all achieve reproductive success $M^{*}(1, Y)$. Hence, residents who play Surrender outperform rare mutants who play Compete if, and only if, $M^{*}(1, Y)>M^{*}(2, Y)$.

Nevertheless, as shown in the last part of the proposition, Compete is always evolutionarily stable, i.e., even when condition (16) holds. To see why, suppose that Compete is the resident strategy. Any Surrendering mutant then almost surely achieves reproductive success equal to 0 , because he is almost surely matched with a resident, who Competes. By contrast, almost all residents get reproductive success $M^{*}(2, Y) / 2{ }^{21}$

\footnotetext{
${ }^{20}$ Evolutionary stability is a static equilibrium concept, akin to that of Walrasian market equilibrium. A dynamic model would be necessary to account for the fact that sons of men who play Compete do not have the same mating success as sons of men who play Surrender. Indeed, to account for such differences one would need a model that tracks how the strategy distribution in generation $t+1$ depends on that in generation $t$.

${ }^{21}$ Note that this result would obtain even under the less stark assumption that playing Surrender against someone playing Compete would give a positive probability of winning, as long as this probability would be below $1 / 2$.
} 
Finally, as indicated in the second part of the proposition, the competition game with constant productive resources admits no evolutionarily stable mixed strategy. To see why, consider a population where some mixed strategy $\zeta \in(0,1)$ is the resident strategy and the mutant pure strategy Compete. An individual who is matched with a resident then does equally well whether he is a resident or a mutant (to see this, note that for a mixed strategy to be ESS it must be such that the residents achieve the same average reproductive success whether they play Surrender or Compete); however, an individual who is matched with a mutant does strictly better if he is a mutant than if he is a resident, since the latter then gets the same reproductive success as the mutant with probability $1-\zeta$ but 0 reproductive success with probability $\zeta$.

In sum, when men compete for women alone there is either one or two evolutionarily stable strategies, depending on the ecology. The assumption that a man's productive resources $(Y)$ do not depend on the number of rounds he competes is, however, restrictive. Indeed, this would require both that the resources a man can ever hope to control are his own physical labor resources, and that these labor resources are unaffected by the amount of competition. In particular, competition cannot entail any risk of physical injury. While examples of male-male competitions satisfying these requirements are conceivable (think of song or poetry contests and chess tournaments), the quest for a model that could help reach a better understanding of the evolution of male-male competition calls for a more general version of the male-male competition game, one in which men compete for both reproductive and productive resources. I now turn to such a model.

\subsection{Competition when both women and productive resources are at stake}

In the competition game with endogenous productive resources, a male who has successfully competed one round has productive resources $\varphi$, which may differ from his initial resources $Y$ (e.g., because of injuries from physical combat, the destruction of tools, the take-over of the opponent's productive resources, etc.). The payoff matrix of this evolutionary game is shown in Figure 2.

\begin{tabular}{|c|c|c|}
\hline & Surrender & Compete \\
\hline Surrender & $M^{*}(1, Y)$ & 0 \\
\hline Compete & $M^{*}(2, \varphi)$ & $\frac{1}{2} M^{*}(2, \varphi)$ \\
\hline
\end{tabular}

Figure 2. Payoffs in the competition game with endogenous productive resources

While many factors can affect the material resources accruing to the winner of a competition, it seems reasonable to put an upper bound on $\varphi$. Specifically, I assume that competition can at most allow the winner to acquire all of the loser's resources, i.e., $\varphi \in[0,2 Y]$. The following results obtain.

Proposition 6. In the competition game with endogenous productive resources:

(i) $\zeta=0$ is evolutionarily stable if and only if: 
(1) either the ecology $\omega$ is such that $M^{*}(1, Y)>M^{*}(2, Y)$ and $\varphi<Y+A$, where $A>0$ is the amount of male resources such that $M^{*}(1, Y)=M^{*}(2, Y+A)$;

(2) or the ecology $\omega$ is such that $M^{*}(1, Y)<M^{*}(2, Y)$ and $\varphi<Y-B$, where $B>0$ is the amount of male resources such that $M^{*}(1, Y)=M^{*}(2, Y-B)$.

(ii) No mixed strategy $\zeta \in(0,1)$ is evolutionarily stable in any ecology $\omega \in \Omega$.

(iii) $\zeta=1$ is evolutionarily stable for any $\omega \in \Omega$ as long as $\varphi \in(0,2 Y]$.

As shown in the proof of the proposition, Surrender is evolutionarily stable if and only if $M^{*}(1, Y)>M^{*}(2, \varphi)$. Since the equilibrium male reproductive success function $M^{*}$ is increasing and continuous in the man's productive resources, this implies that Surrender is evolutionarily stable if either (1) the ecology is such that Surrender is evolutionarily stable when productive resources are constant and winning one round of competition does not enhance productive resources by too much, or (2) the ecology is such that Surrender is not evolutionarily stable for constant resources but winning one round of competition entails a large enough drop in productive resources.

The astute reader will have noticed that while Compete is evolutionarily stable for any positive $\varphi>0$, it no longer is so for $\varphi=0$. To see why, recall that Compete is evolutionarily stable against Surrender if there exists some threshold value $\bar{\varepsilon}>0$ such that for all $\varepsilon \in(0, \bar{\varepsilon})$, the average reproductive success of men playing Compete, when this is the resident strategy present in a share $\varepsilon$ of the population, strictly exceeds that of men playing Surrender:

$$
(1-\varepsilon) \cdot \frac{M^{*}(2, \varphi)}{2}+\varepsilon \cdot M^{*}(2, \varphi)>\varepsilon \cdot M^{*}(1, Y) \text {. }
$$

As long as resource depletion is not complete (i.e., for any $\varphi>0), \frac{M^{*}(2, \varphi)}{2}>0$ and the inequality holds for $\varepsilon$ close enough to zero, even if $M^{*}(1, Y)>M^{*}(2, \varphi)$ (to see this, set $\varepsilon=0$, and note that both the left-hand and the right-hand side is continuous in $\varepsilon$ ). By contrast, when Compete entails full resource depletion $(\varphi=0)$, average reproductive success is nil for men playing Compete while it is strictly positive for men playing Surrender.

In sum, then, on top of ecological factors, any factors (e.g., technological and/or institutional factors) that affect the material resources accruing to the winner of a competition should also be expected to impact the evolutionary stability of non-competitive males. Remarkably, however, these factors have no impact on the evolutionary stability of competitive males: in all the settings analyzed above Compete is an evolutionarily stable strategy. This is true even though any population would be better off as a whole if males were non-competitive, since female reproductive success is strictly decreasing in the polygyny rate (this follows from Proposition 4 and the assumption that winning a competition cannot more than double a man's productive resources $(\varphi \leq 2 Y) .{ }^{22}$ In other words, in any setting where both Surrender and Compete are evolutionar-

\footnotetext{
${ }^{22}$ The only setting in which female reproductive success is not strictly decreasing in the polygyny rate is when pater-
} 
ily stable, evolution could have led either to the (efficient) absence of male-male competition, or to the (inefficient) presence of male-male competition, where efficiency is measured in terms of female reproductive success.

In the next subsection I extend the model to allow for an ubiquitous aspect of human life that will be seen to sometimes render the strategy Compete evolutionarily unstable.

\subsection{The effects of relatedness on male-male competition}

So far, the analysis has disregarded an important and ubiquitous feature of human societies, namely, the fact that our ancestors lived in groups of small size, which extended beyond the nuclear family and between which there was limited migration. ${ }^{23}$ A key implication of such population structure is that mutants, even when rare, face a higher probability of interacting with mutants than residents do, even absent any ability of individuals to choose the individuals with whom they interact. In the biology literature, this effect is usually quantified by the coefficient of relatedness (Wright, 1931), which measures the probability that interacting individuals share a common ancestor. In order to extend the model in this direction I adopt the formalization proposed by Bergstrom (2003) (see also Grafen, 1979, Alger and Weibull, 2013, and Jensen and Rigos, 2018). ${ }^{24}$

Thus, consider the evolutionary game analyzed in the preceding subsection, and denote the resident strategy by $\zeta \in[0,1]$ and the mutant strategy by $\zeta^{\prime} \in[0,1], \zeta^{\prime} \neq \zeta$, the latter being present in a (small) share $\varepsilon>0$ of the population. The assortment function $\sigma:(0,1) \rightarrow[-1,1]$ maps to each mutant population share $\varepsilon \in(0,1)$ the difference between the probability for a resident to be matched with a resident and a mutant to be matched with a resident (the assortment function is the same for all $\left.\left(\zeta, \zeta^{\prime}\right) \in[0,1]^{2}\right)$ :

$$
\sigma(\varepsilon)=\operatorname{Pr}[\zeta \mid \zeta, \varepsilon]-\operatorname{Pr}\left[\zeta \mid \zeta^{\prime}, \varepsilon\right]
$$

In the special case of uniform random matching, there is no difference, i.e., $\sigma(\varepsilon)=0$ for all $\varepsilon \in$ $(0,1)$. I assume that both conditional probabilities $\operatorname{Pr}[\zeta \mid \zeta, \varepsilon]$ and $\operatorname{Pr}\left[\zeta \mid \zeta^{\prime}, \varepsilon\right]$ are continuous in $\varepsilon$, and denote by $\sigma_{0}$ the limit of the assortment function $\sigma$ as the share of mutants tends to zero: $\sigma_{0} \equiv \lim _{\varepsilon \rightarrow 0} \sigma(\varepsilon)$. Noting that $\lim _{\varepsilon \rightarrow 0} \operatorname{Pr}[\zeta \mid \zeta, \varepsilon]=1$ (because the population is infinitely large), it is clear that $\lim _{\varepsilon \rightarrow 0} \sigma(\varepsilon)=1-\lim _{\varepsilon \rightarrow 0} \operatorname{Pr}\left[\zeta \mid \zeta^{\prime}, \varepsilon\right]=\lim _{\varepsilon \rightarrow 0} \operatorname{Pr}\left[\zeta^{\prime} \mid \zeta^{\prime}, \varepsilon\right]$. In other words, $\sigma_{0}$ is also the

nal investment comes in the form of a purely public good $(\alpha=0)$ and all the productive resources are acquired by the winning male $(\varphi=2 Y)$. It is then constant in the polygyny rate.

${ }^{23}$ Admittedly, migration rates are higher in some populations than in others, and they can be quite high (see, e.g., Kramer, Schacht, and Bell, 2017). However, what matters for the discussion at hand is that less than $100 \%$ of men leave their natal group.

${ }^{24}$ While this formalization is less fine-grained than that obtained in the classic island model in evolutionary biology (see, e.g., Cavalli-Sforza and Bodmer, 1971, Frank 1998, Rousset 2004, Hartl and Clark, 2007), it is sufficient for my purposes. For a recent adoption of the island to preference evolution in srategic interactions, see Alger, Weibull, and Lehmann (2020). 
probability that a mutant is matched with another mutant in the limit as the share of mutants tends to 0 . It follows that $\sigma_{0} \in[0,1]$. For my purposes I will say that relatedness is present when $\sigma_{0}>0$ and absent when $\sigma_{0}=0$. The latter case having been analyzed above, I here assume $\sigma_{0}>0$. Letting

$$
\tilde{\sigma}_{0} \equiv \frac{M^{*}(2, \varphi)}{2 \cdot M^{*}(1, Y)}
$$

the following results obtain.

Proposition 7. In the competition game with endogenous productive resources and relatedness:

(i) the set of parameter values for which $\zeta=0$ is evolutionarily stable when $\sigma_{0}=0$ (see Proposition 6 ) is a proper subset of the set of parameter values for which $\zeta=0$ is evolutionarily stable when $\sigma_{0} \in(0,1]$.

(ii) No mixed strategy $\zeta \in(0,1)$ is evolutionarily stable in any ecology $\omega \in \Omega$.

(iii) $\zeta=1$ is evolutionarily stable if $\sigma_{0}<\tilde{\sigma}_{0}$ while it is not evolutionarily stable if $\sigma_{0}>\tilde{\sigma}_{0}$.

Comparing these results to those reported for the setting without relatedness $\left(\sigma_{0}=0\right)$ in Proposition 6, it is clear that relatedness works in favor of the Surrender strategy and against the Compete strategy. The effect is twofold.

First, relatedness expands the set of parameter constellations for which Surrender is evolutionarily stable. Compared to the setting without relatedness, competition can entail a larger increase in productive resources $\varphi-Y$ without threatening the stability of Surrender in ecologies for which Surrender is evolutionarily stable under constant resources; likewise, a smaller drop in productive resources $Y-\varphi$ due to competition is sufficient to render Surrender evolutionarily stable in ecologies for which Surrender is evolutionarily stable under constant resources.

Second, and in stark contrast with Propositions 5 and 6, Compete fails to be evolutionarily stable if relatedness is pronounced enough. The threshold value for $\sigma_{0}$ (see (19)) is the ratio of male reproductive success obtained if all males Compete $\left(M^{*}(2, \varphi) / 2\right)$ to that obtained if all males Surrender $\left(M^{*}(1, Y)\right)$. To understand why, suppose that Compete is the resident strategy and Surrender the mutant one. A necessary condition for Compete to be evolutionarily stable against Surrender is that the average reproductive success of residents be at least as large as that of mutants, when the share of mutants tends to zero, i.e.:

$$
\frac{M^{*}(2, \varphi)}{2} \geq\left(1-\sigma_{0}\right) \cdot 0+\sigma_{0} \cdot M^{*}(1, Y)
$$

The right-hand side shows that, even in a population where essentially all individuals Compete, a vanishingly rare mutant, who Surrenders, faces a positive probability of interacting with another mutant, thereby being able to settle down with his teenage girlfriend. If Compete entails an inefficiency, i.e., if $M^{*}(2, \varphi) / 2$ falls short of $M^{*}(1, Y)$, and if rare mutants have a sufficiently strong tendency to interact with each other, the necessary condition (20) is violated: the inefficient strategy Compete then cannot withstand the invasion of the more efficient strategy Surrender. 
Thus, whenever the threshold value $\tilde{\sigma}_{0}$ for $\sigma_{0}$ falls short of 1 , Compete fails to be evolutionarily stable for any $\sigma_{0} \in(\tilde{\sigma}, 1]$. The ratio in (19) depends both on the ecology $(\omega)$ and on the effect of competition on productive resources $(\varphi-Y)$. For any given effect of competition on productive resources, $\tilde{\sigma}$ is increasing in the effect of adding a second wife under constant productive resources $\left(M^{*}(2, Y)-M^{*}(1, Y)\right)$. And for any given ecology, $\tilde{\sigma}$ is increasing in the productive resources that competition bestows on the winner $(\varphi)$.

\subsection{Female choice}

Clearly, the assumption that women passively accept to join the household allocated to them through the male-male competition is highly restrictive. Here I extend the model to allow for female choice, thus accounting for female preferences.

Following the male-male competition stage, women can choose between accepting the household formation (as above) and not accepting it, in which case one woman settles down with the winner and the other with the loser; I assume that the winner's teenage sweetheart (the "first wife") gets to choose first. The key issue is how the male-male competition affects the men' productive resources. Let $\varphi-\Delta$ denote the productive resources accruing to the loser, where $\Delta>0$. Assuming, as above, that a winner can garner at most an amount $Y$ by winning a contest, $\Delta \in(0,2 Y)$ : if $\Delta$ is close to $2 Y$, the man who loses a contest also loses almost all his productive resources. Clearly, in this extreme case a woman would prefer to marry bigynously with the winner (who has productive resources close to $2 Y$ ) than monogamously with the loser (who has productive resources close to zero). The following proposition follows immediately from this observation together with the fact that the female equilibrium reproductive success function $F^{*}$ is continuous and increasing in the man's productive resources, and is decreasing in $k$ (see the last part of Proposition 4).

Proposition 8. If women can choose a husband after the male-male competition stage:

(i) in populations where all men Surrender, each woman accepts to marry monogamously;

(ii) in populations where all men Compete, the loss of productive resources $\Delta$ takes a value in $(0,2 Y)$ depending on the nature of the contest, and there exists $\tilde{\Delta} \in(0,2 Y)$ such that the second wife prefers bigynous marriage with the winner to monogamous marriage with the loser iff $\Delta>\tilde{\Delta}$.

Faced with the fait-accompli of the male-male competition, female reproductive success is higher under bigyny than under monogamy whenever it generates a large enough difference between the winner's and the loser's productive resources. ${ }^{25}$ Overall, the proposition shows that the assumption that women passively accept the household composition determined by the male-male

\footnotetext{
${ }^{25}$ This is in line with the polygyny threshold model (Orians, 1969), and the ensuing literature on polygyny arising from male heterogeneity (see the references in the introduction). The advantage is that here the heterogeneity among men is endogenously determined through male-male competition.
} 
competition is innocuous in settings where (under the passivity assumption) either Surrender is evolutionarily stable, or Compete is evolutionarily stable and the competition gives rise to enough heterogeneity in productive resources between the men $(\Delta>\tilde{\Delta})$.

It remains to be seen whether female choice alters the results in settings where under female passivity Compete is evolutionarily stable, but where both the winner and the loser would end up marrying monogamously upon competing when the passivity assumption is lifted. In other words, is Compete evolutionarily stable in the male-male competition game when the contest affects only the productive resources? The payoff matrix of this game is shown in Figure 3. The next proposition treats this case, allowing for relatedness.

\begin{tabular}{|c|c|c|}
\hline & Surrender & Compete \\
\hline Surrender & $M^{*}(1, Y)$ & 0 \\
\hline Compete & $M^{*}(1, \varphi)$ & $\frac{1}{2}\left[M^{*}(1, \varphi)+M^{*}(1, \varphi-\Delta)\right]$ \\
\hline
\end{tabular}

Figure 3. Payoffs in the competition game which bestows productive resources $\varphi \in(0,2 Y]$ on the winner and $\varphi-\Delta>0$ on the loser, with female mate choice and $\Delta<\tilde{\Delta}$

Letting

$$
\hat{\sigma}_{0} \equiv \frac{M^{*}(1, \varphi)+M^{*}(1, \varphi-\Delta)}{2 \cdot M^{*}(1, Y)},
$$

the following result obtains.

Proposition 9. Consider the competition game where the winner's productive resources exceeds those of the loser by $\Delta \in(0, \tilde{\Delta})$. Under female choice, Compete is evolutionarily stable if the coefficient of relatedness $\sigma_{0}$ is smaller than $\hat{\sigma}_{0}$ while it is not evolutionarily stable if $\sigma_{0}>\hat{\sigma}_{0}$.

In other words, the qualitative nature of the condition for Compete to be evolutionarily stable obtained under female passivity is preserved under female choice. Interestingly, however, relatedness can be less effective at preventing male-male competition with than without female choice. For example, in the extreme case where $\Delta \approx 0$ and $\varphi=Y$, the threshold value $\hat{\sigma}_{0} \approx 1$. This is because when female choice leads to monogamy, it prevents the inefficiency inherent to bigyny from arising.

The model disregards the possibility that women develop a taste or distaste for more competitive men. Analysis of the evolution of such preferences would require a different model, which would include a detailed analysis of the matching process in the marriage market (and in particular of how conflict between women with the same preferences over male types would be resolved). ${ }^{26}$ Likewise, the model abstracts from sources of heterogeneity among men beyond their

\footnotetext{
${ }^{26} \mathrm{An}$ interesting challenge for such a generalization will be to determine whether one should also allow for the evolution of male preferences over female preferences over male competitiveness, and so forth.
} 
degree of competitiveness and productive resources, such as as strength, speed, intelligence etc. These factors should certainly be should be taken into account in future research, which may thus examine the interaction of female preferences over such attributes and the forces studied here.

\subsection{Summing up}

Taken together, the results reported above suggest that-absent inter-generational transmission of wealth and unbalanced sex ratios-in human history high levels of male competitiveness would have been consistent with evolution by natural selection only in times and places where the following three conditions were met simultaneously.

1. The ecology $\omega$ must be generous enough for the male adult household member not to be required to engage heavily in provisioning food (or some other rival good) to his offspring.

2. In the least generous ecologies the competition must increase the winner's productive resources enough for the women to prefer bigynous marriage with the winner than monogamous marriage with the loser.

3. Relatedness between competing males must be low enough.

Importantly, it is the combination of these three factors, rather than each factor alone, that is necessary for high levels of competitiveness to be compatible with evolution by natural selection.

The next section discusses the ramifications of these insights for our understanding of the evolution of marriage systems in human history. In particular, I argue that the combination of the three factors mentioned above may well have been uncommon over the course of human history, suggesting that polygynous marriage may have been driven mostly by forces omitted in this model (such as the ability to transmit wealth across generations, which for any given degree of competitiveness induces heterogeneity in men's ability to win a contest).

\section{Discussion}

The theoretical model analyzed above provides a rich set of predictions. While it is beyond the scope of this paper to conduct a formal empirical analysis (which would necessitate fine-grained data that would allow to control for factors not considered in this model), in this section I discuss the model predictions in light of a few important sources of variation across human subpopulations over space and time. In the model a population is fully described by the following three components: the ecology $(\omega \in \Omega)$, the effect of competition on male productive resources $(Y-\varphi)$, and the coefficient of relatedness $\left(\sigma_{0} \in[0,1]\right)$. I first discuss in general how the ecology and the coefficient of relatedness may have varied across space and time, and then propose a structured comparison of different human sub-populations through history, a comparison which also 
discusses how technological change may have altered the effect of competition on male productive resources.

Prior to the industrial revolution, the vast majority of people were self-sustaining farmers, horticulturalists, pastoralists, or hunter-gatherers. As such, their livelihoods depended to a large extent on the climatic, geological, and ecological conditions in their local environment. Since Homo sapiens had colonized all continents except Antarctica already in pre-neolithic times, in terms of the model this means that different human sub-populations faced a rich set $\Omega$ of different ecologies both in pre- and in post-neolithic times. Sources of variation would have included the marginal returns to male and female food production efforts, the nutritional value of local food sources, environmental hazards—such as natural disasters, predators, and attacks by other groups-some of which it was possible to protect against, etc. Arguably, it is reasonable to assume that in pre-industrial times (and using again the term "ecology" as defined in the model): (1) at any point in time there would have been significant spatial variation between the ecologies $\omega \in \Omega$ faced by human sub-populations; and (2) in any given location, sub-populations that adopted agriculture would have experienced a substantial change in the ecology $\omega$ compared to the preneolithic one, because of changes in the food production technology and in the food sources, and possibly also in the prevalence of environmental hazards; there would nonetheless still be spatial variation in the ecologies faced by sub-populations having adopted agriculture, due to variation in geological and climatic conditions.

In the model the ecology matters because it affects the quantity-quality trade-off that men face. Hence, the model predicts that male reproductive success as a function of the number for wives may have been quite different in a climate with long and harsh winters than in a tropical or sub-tropical climate. Existing studies do suggest a significant impact of the ecology (as defined in the model, i.e., incorporating all aspects of food and care production) on reproductive success, even for populations living in the same natural environment. For example, a comparison of agriculturalists and nomadic herders in Northern Finland born between 1641 and 1884 shows that the agriculturalists had, on average, a significantly higher number of offspring, about 0.3 children more per (monogamous) family, while there was no significant difference in mortality prior to adulthood (Helle et al., 2014). A study of contemporary Pumé, an indigenous group in Venezuela, revealed that women among the horticulturalist river Pumé have a significantly larger number of children who survive to adulthood than the foraging savannah Pumé (Kramer and Greaves, 2007).

Turning to relatedness, the key driver is migration. To see this, consider first a population in which each individual would migrate on his/her own from the group into which (s)he was born to a randomly chosen group of people anywhere else in the world. In such a population, the probability of interacting with individuals sharing a recent common ancestor would be close to zero. By contrast, if only some individuals disperse from their natal group, or if all disperse to a place close to the birthplace, the probability of interacting with individuals sharing a recent common 
ancestor is positive. Clearly, human sub-populations are best described by the second scenario. What matters for the model is the relatedness among men, and thus men's dispersal. While comprehensive historical data is simply missing, some studies do provide some insights. For example, in their study of dispersal of individuals born in Finland between 1749 and 1880, Nitsch, Lummaa, and Faurie (2016) find that around 20\% of all individuals who survived to the age of 15 moved out of their birth parish, and that the average distance between the birth parish and the destination parish was only $65 \mathrm{~km}$. While these numbers were similar for men and women, a key difference between the sexes was that among the individuals who dispersed, women were almost twice as likely to move to the spouse's birth parish, implying a higher coefficient of relatedness among young men than among young women. ${ }^{27}$

With these factors in mind, I structure the discussion of the model predictions around two qualitatively different types of society, according to the impact of competition on a man's productive resources. In this discussion I will assume that $\varphi=b c Y$, where $c \in[0,1]$ and $b \in[1,2]$ are parameters representing, respectively, the resource depletion and resource accumulation associated with competition.

\subsection{Competition without resource accumulation: pre-neolithic societies}

Imagine a society where the only labor resources a man can ever hope to control are his own physical labor resources; that is, slavery is not an option, and no or little accumulation of material resources beyond those necessary for survival (such as basic tools and shelter) is possible. In such a society, the only prize that men compete for is access to women. If competition, moreover, entails some risk of physical injury, then labor resources may be expected to decline with competition, or at least not increase. In terms of the model, in this society, the accumulation factor $b$ would be equal to or close to 1 , while the depletion factor $c$ would be small enough for competition to entail resource depletion, or at least not any resource accumulation $(b c \leq 1)$.

Arguably, this scenario appears plausible for many human societies prior to the neolithic revolution. Absent agricultural production, any group of Homo sapiens had to rely on the food provided by its surrounding natural environment, rendering individual land ownership irrelevant. In most places climatic conditions further restricted the ability to store any food surpluses. While it is challenging to evaluate the extent to which men provisioned children with food in pre-neolithic times, among modern hunter-gatherers and horticulturalists men heavily engage in such provisioning, at least in some societies (Kaplan et al., 2001). ${ }^{28}$ Moreover, since Homo sapiens mastered

\footnotetext{
${ }^{27}$ This would be in line with Murdock's ethnographic atlas, according to which virilocality (the tendency for women to move to the husband's locality) has been more prevalent than uxorilocality (the tendency for men to move to the wife's locality): out of 1267 societies, 692 were classified as mainly virilocal and only 305 as mainly uxorilocal. This classification has been revisited in past years, however; see, e.g., Kramer and Greaves (2011) an references therein.

${ }^{28}$ Moreover, a recent study suggests that male provisioning had already evolved when Homo sapiens appeared (Alger et al., 2020; see, in particular, the discussion of the paleontological and archeological evidence).
} 
the production and use of lethal tools such as axes and spears, contests between men could entail significant physical injury.

Viewed through the lens of my model, it thus appears plausible that pre-neolithic societies would have evolved to have non-competitive men and monogamous unions: challenging ecological conditions coupled with the absence of resource accumulation $(b c \leq 1)$ could have been such that male reproductive success was higher with one than with two wives $\left(M^{*}(1, Y)>M^{*}(2, \varphi)\right)$, thus rendering the Surrender strategy evolutionarily stable, i.e., non-competitive men compatible with evolution by natural selection. If, moreover, relatedness among young men was high enough, the Compete strategy would not have been evolutionarily stable (see Proposition 7). Such a conclusion would be compatible with evidence from traditional societies that have been observed over the past few hundred years: an overwhelming majority of unions in these societies are monogamous (see the references in the second paragraph of the introduction). ${ }^{29}$

\subsection{Competition with resource accumulation: agricultural societies}

In terms of the model, the advent of agriculture brought about two key changes. First, in any given location on Earth, the adoption of agriculture likely induced a change in some parameters of the ecology $\omega=(b, \phi, \sigma, \lambda, \tau, X, \alpha, \beta, \gamma, \theta, \rho)$, especially the marginal returns to male and female labor in food production (the parameters $\gamma, \theta$, and $\rho$ ), but potentially also the extent to which the non-food contribution of the father was a rival good (the parameter $\alpha$ ). Second, food production became based on transferable assets such as land and farmed animals. ${ }^{30}$ In the model the former change would typically have induced changes in the intra-household division of labor, while the latter change meant that, contrary to most pre-agricultural societies, the control of productive assets now also became a stake of male-male competition. I argue that in the model these two changes may have had either qualitatively similar or opposite effects on male-male competition.

First, the type of crops and animal husbandry that a sub-population adopted, together with the tools at hand as well as the local geological and climatic conditions, would all have mattered for the precise changes in the ecology $\omega$ that the adoption of agriculture would have induced. In particular, these factors could have led men to become more or less involved in the production of food (and other non-rival goods) than in pre-agricultural times. For example, men's involvement in food production may have intensified more in societies that adopted the plough than in societies that didn't (Goody, 1976). Ceteris paribus, the changes in $\omega$ due to the transition to agriculture could thus have either strengthened or weakened the evolutionary stability of the Sur-

\footnotetext{
${ }^{29}$ There are some interesting exceptions, however. For example, polygyny is quite common among the Yanomamö in Venezuela, although the men who marry polygynously are not wealthier than those who do not (Hames, 1996). This is consistent with the evolutionary stability of the Compete strategy even in societies where winners do not take over the productive resources of their adversaries.

${ }^{30}$ Recently, a model proposed by Bowles and Choi (2019) suggests that the property rights institution developed early on during the Neolithic transition.
} 
render strategy.

Second, in an extreme variant of a society in which productive assets are seizable, the winner's productive resources double $(b c=2)$. If the protection that the man provides to the children in his household is not fully rival $(\alpha<1)$ the reproductive success of a man with two wives is more than twice as large as that of a man in a monogamous union, while if it is fully rival $(\alpha=1)$ his reproductive success is exactly twice as large. In any event, in such a society only Compete would be evolutionarily stable, because the coefficient of relatedness required to prevent this would be excessively high $(\tilde{\sigma} \geq 1$, see equation (19)). A doubling of productive resources does not appear historically relevant, however: since productive resources would have included the man's own labor, a doubling of productive resources would have required the losing man to become the winner's slave. Hence, the most likely scenario is that in the early agricultural societies the winner would see his productive resources increase but not double $(1<b c<2)$. The model predicts that this would have tilted the balance in favor of the Compete strategy.

Arguably, however, the advent of metallurgy further affected the trade-offs. Indeed, significant technological advances followed in the footsteps of the agricultural life-style. In particular, weapons became more sophisticated and lethal, especially with the advent of metallurgy. As a result, men would have had to sacrifice an increasing amount of resources to make or acquire weapons and adequate protective gear. In the model such technological advances correspond to a decrease in the depletion parameter $c$ over time. Depending on the accumulation parameter $b$, this development could have induced a negative net effect of competition on productive resources (i.e., $b c<1$ ). Hence, the advent of metallurgy should have tilted the balance back in favor of the Surrender strategy.

\subsection{Three major paths?}

In sum, my findings suggest that the evolution of the prevalence of polygynous unions in any given human sub-population is specific to the local constraints it faced together with the technology it adopted for food extraction (the "ecology" $\omega$ in the model), the seizability and/or vulnerability of productive resources due to male-male competition (the difference $\varphi-Y$ in the model), and the extent to which competing males share a recent common ancestor (the relatedness parameter $\sigma_{0}$ ). In particular, the discussion above indicates that the transition to an agricultural life-style would not necessarily have given rise to more competitive men and polygyny, especially in ecologies in which men chose to engage substantially in the production of food (and/or some other rival goods). ${ }^{31}$ More generally, the theoretical findings and the discussion above point to three major historical development paths for human sub-populations, each path being defined by its most common marriage system in pre-neolithic times, in neolithic times, and during the

\footnotetext{
${ }^{31}$ Note that any effects of storability of wealth is absent from my model since there is no transfer of wealth between generations. Such storability could thus have had effects on top of those discussed here.
} 
bronze and iron ages.

- Path 1: Monogamy - Polygyny - Polygyny

- Path 2: Monogamy - Polygyny - Monogamy

- Path 3: Monogamy - Monogamy - Monogamy

In accordance with the discussion in subsection 4.1, all paths have monogamy as the main marriage system in pre-neolithic times. ${ }^{32}$ Due to the seizability of productive assets-especially land-that it implied, the adoption of agriculture would have led to significant levels of polygyny, but only in societies where ecological conditions were such that men did not need to engage heavily in the production of food (Paths 1 and 2). Hence, polygyny would not have arisen in human sub-populations which either did not adopt agriculture or which did transition to agriculture but faced harsh conditions that rendered strong male involvement in food production necessary (Path 3). 33

Finally, among the populations that adopted agriculture, a distinction can be made between those that later adopted metallurgy and those that didn't. Those that didn't should not have experienced any change. Those that did develop metallurgy may-due to the lethality of weaponseither have continued to be largely monogamous (Path 3), transitioned back from polygyny to monogamy if the relatedness among competing males was high enough (Path 2), or remained polygynous if the relatedness among competing males was low enough (Path 1). ${ }^{34}$

Remark. While I have focused the discussion on Homo sapiens, the model arguably applies to any species in which males provide parental care. An obvious illustration that comes to mind is birds, in which "monogamy is ten times more common [...] than in mammals" (De Waal and Gavrilets, 2013). Since male birds tend to provision their hatchlings with food, this observation appears to be consistent with the model predictions. However, a rigorous test of whether paternal provisioning of food (or some other rival good) is correlated with monogamy across species would require a dataset that includes numerous other factors that evolutionary biologists have identified as potential ultimate drivers of monogamy (see, e.g., Klug, 2018).

\footnotetext{
${ }^{32}$ This is in line with Fortunato (2011), who concludes based on a phylogenetically controlled study that monogamy likely was a common marriage form among early Indo-European peoples, prior to the advent of state-like social structures.

${ }^{33}$ The finding by Barber (2008) that polygyny is found mostly in tropical countries with plenty of arable land is consistent with this hypothesis. See also Lee and Whitbeck (1990) and references therein for evidence of correlation between polygyny and female labor investment in food production.

${ }^{34}$ Taken together, these three paths should have induced the share of men who were successful at reproducing to be the lowest in neolithic societies. This is consistent with the drop in Y-chromosome diversity for the period between approximately $10 \mathrm{kya}$ and $6 \mathrm{kya}$, and the subsequent rise in this diversity, as inferred from contemporary data by Karmin et al. (2015). These paths would further be consistent with the relatively low levels of physical violence observed between men, and which has been the subject of many theories (see the overview by Seabright, 2004); the theory proposed here arguably sheds new light on this question.
} 


\section{Conclusion}

Biological reproduction is the central driver of natural selection, and in a sexually reproducing species such as ours the competition for reproductive resources is fiercer for men than for women. One should therefore expect evolutionary forces to have shaped the inclination to compete differently for men and for women. The model proposed here contributes to the understanding of these forces, by examining the competition between men for access to both reproductive and productive resources, when the driver of behavior is biological reproductive success. The analysis focuses on three ubiquitous features of human history that arguably had first-order effects on the incentives for men to engage in competitive strategies. First, local ecological factors, such as the effort needed to produce food and the returns from the father's and the mother's protection and care, was key to reproductive success in all pre-industrial times. Second, the advent of agriculture brought about a drastic change in the incentives to compete; however, by contrast to the storability of wealth that is often put forward as being the key effect of agriculture, I focus on the seizability of productive assets that the advent of agriculture brought about. Finally, the model also incorporates the fact that in all human societies there is limited migration; such limited migration implies that competing men would typically have been related. The central insight is that in this setting high levels of male competitiveness are consistent with evolution by natural selection only if (a) the ecology in which the population evolved would have been generous enough for males to supply little or no food to their children, (b) competing would not have been too costly in terms of productive resources, and (c) relatedness among males would have been low enough.

While the model innovates in several respects compared to the existing literature on the evolution of marriage systems, it disregards some arguably relevant aspects, which should be accounted for in future research. First, it would be interesting to extend the model to allow for the ability to transmit wealth across generations (Bergstrom, 1994a, Lagerlöf, 2005). Second, while ecological constraints and relatedness may have led men to refrain from competing against each other within groups, as suggested by my model, competition between groups of males for women and productive resources may have arisen instead (Henrich, Boyd, and Richerson, 2012). This hypothesis finds support in the empirical analyses of historical Y-chromosome diversity by Balaresque et al. (2015) and Zeng, Aw, and Feldman (2018). Third, and as pointed out by Betzig (1992), "marriage in Rome was monogamous; mating was polygynous". Hence, future analyses should also examine the consequences of sexual infidelity for the evolution of male competitiveness. ${ }^{35}$ Finally, in the model I take the degree of competitiveness to be transmitted "vertically", from father to son. Future research may examine if the results are robust to a mix of vertical and oblique transmission, that would be relevant if male competitiveness is a cultural rather than a biological trait (Bisin and Verdier, 2001).

\footnotetext{
${ }^{35}$ For further inspiration on this and related topics, see Hrdy (1999) and Fisher (2016) and references therein.
} 
The theory presented here is arguably also relevant beyond the question of how marriage systems evolved in human societies. Indeed, it adds the competition for mates as an ultimate driver of willingness to outperform others, to the literature on the evolutionary foundations of interdependent preferences (Koçkesen, Ok, and Sethi, 2000a,b, Heifetz, Shannon, and Spiegel, 2007, Alger, Weibull, and Lehmann, 2020). Since this literature has hitherto neglected biological differences between men and women, this paper contributes insights to aspects of the evolution of competitiveness that are specific to men. A similar approach could be used to investigate aspects of the evolution of competitiveness specific to women. This line of research could thus help understand and interpret the evidence suggesting that men and women differ in their willingness to outperform others (e.g., Gneezy, Niederle, and Rustichini, 2003, Datta Gupta, Poulsen, and Villeval, 2013, Apicella and Dreber, 2015; see also Croson and Gneezy, 2009, and Bertrand, 2011, for surveys).

\section{Appendix: Proofs}

\subsection{Proof of Proposition 1}

I first prove that any Nash equilibrium strategy profile is female-symmetric. To this end, I assume, by contradiction, that there exists a Nash equilibrium strategy profile according to which $k_{1}$ women play strategy $\left(n_{1}, x_{1}\right), k_{2}=k-k_{1}$ women play strategy $\left(n_{2}, x_{2}\right) \neq\left(n_{1}, x_{1}\right)$, and the man plays strategy $y$. Defining

$$
a(n)=\max \{0,1-b n\},
$$

the necessary conditions for this to be an equilibrium are:

$$
\left(n_{1}, x_{1}\right) \in \arg \max _{(n, x) \in[1,+\infty) \times[0, X]} n \cdot a(n) \cdot s\left(n, x,\left(n_{1}, n_{2}\right)^{\left(k_{1}-1, k_{2}\right)}, y\right),
$$

and

$$
\left(n_{2}, x_{2}\right) \in \arg \max _{(n, x) \in[1,+\infty) \times[0, X]} n \cdot a(n) \cdot s\left(n, x,\left(n_{1}, n_{2}\right)^{\left(k_{1}, k_{2}-1\right)}, y\right),
$$

where $\left(n_{1}, n_{2}\right)^{\left(k_{1}-1, k_{2}\right)}$ denotes the $(k-1)$-dimensional vector whose first $k_{1}-1$ components equal $n_{1}$ and the remaining $k_{2}$ components equal $n_{2}$, and $\left(n_{1}, n_{2}\right)^{\left(k_{1}, k_{2}-1\right)}$ the $(k-1)$-dimensional vector whose first $k_{1}$ components equal $n_{1}$ and the remaining $k_{2}-1$ components equal $n_{2}$. Furthermore, the strategies $\left(n_{1}, x_{1}\right)$ and $\left(n_{2}, x_{2}\right)$ must yield the same reproductive success, i.e.:

$$
n_{1} \cdot a\left(n_{1}\right) \cdot s\left(n_{1}, x_{1},\left(n_{1}, n_{2}\right)^{\left(k_{1}-1, k_{2}\right)}, y\right)=n_{2} \cdot a\left(n_{2}\right) \cdot s\left(n_{2}, x_{2},\left(n_{1}, n_{2}\right)^{\left(k_{1}, k_{2}-1\right)}, y\right)
$$


Without loss of generality, assume that $n_{1}>n_{2}$. Then a woman who deviates from $\left(n_{1}, x_{1}\right)$ to $\left(n_{2}, x_{2}\right)$ achieves reproductive success

$$
n_{2} \cdot a\left(n_{2}\right) \cdot s\left(n_{2}, x_{2},\left(n_{1}, n_{2}\right)^{\left(k_{1}-1, k_{2}\right)}, y\right) \text {, }
$$

where

$$
\begin{aligned}
s\left(n_{2}, x_{2},\left(n_{1}, n_{2}\right)^{\left(k_{1}-1, k_{2}\right)}, y\right)= & {\left[\frac{Y-y}{\left[\left(k_{1}-1\right) n_{1}+\left(k_{2}+1\right) n_{2}\right]^{\alpha}}\right]^{\lambda} . } \\
& \cdot\left(\frac{X-\phi x_{2}}{n_{2}^{\beta}}\right)^{\sigma} \cdot\left[\frac{\rho \gamma x_{2}}{n_{2}}+\frac{\theta y}{\left(k_{1}-1\right) n_{1}+\left(k_{2}+1\right) n_{2}}\right]^{\tau} .
\end{aligned}
$$

Since $n_{1}>n_{2}$, this is strictly greater than

$$
s\left(n_{2}, x_{2},\left(n_{1}, n_{2}\right)^{\left(k_{1}, k_{2}-1\right)}, y\right)=\left[\frac{Y-y}{\left(k_{1} n_{1}+k_{2} n_{2}\right)^{\alpha}}\right]^{\lambda} \cdot\left(\frac{X-\phi x_{2}}{n_{2}^{\beta}}\right)^{\sigma} \cdot\left[\frac{\rho \gamma x_{2}}{n_{2}}+\frac{\theta y}{k_{1} n_{1}+k_{2} n_{2}}\right]^{\tau} .
$$

Together with (25), this in turn implies

$$
n_{2} \cdot a\left(n_{2}\right) \cdot s\left(n_{2}, x_{2},\left(n_{1}, n_{2}\right)^{\left(k_{1}-1, k_{2}\right)}, y\right)>n_{1} \cdot a\left(n_{1}\right) \cdot s\left(n_{1}, x_{1},\left(n_{1}, n_{2}\right)^{\left(k_{1}-1, k_{2}\right)}, y\right)
$$

which contradicts (23). A similar argument proves that there exists no Nash equilibrium strategy profile in which women employ three or more different fertility strategies. I conclude from this that any equilibrium of the household game is such that all women have the same fertility. Together with strict concavity of $s$ in its second argument $(x)$, this implies that the womens' equilibrium time allocations are also identical.

Having thus proved that any Nash equilibrium strategy profile is female-symmetric, I proceed to characterize the set of Nash equilibria. Any female-symmetric strategy profile $\left(n^{*}, x^{*}, y^{*}\right)$ is a Nash equilibrium strategy profile if and only if

$$
\left\{\begin{array}{c}
n^{*} \in \arg \max _{n \in[1,+\infty)} F\left(n, x^{*},\left(n^{*}\right)^{(k-1)}, y^{*}\right) \\
x^{*} \in \arg \max _{x \in[0, X]} F\left(n^{*}, x,\left(n^{*}\right)^{(k-1)}, y^{*}\right) \\
y^{*} \in \arg \max _{y \in[0, Y]} M\left(\boldsymbol{n}^{*}, \boldsymbol{x}^{*}, y\right),
\end{array}\right.
$$

where $\left(n^{*}\right)^{(k-1)}$ denotes the $(k-1)$-dimensional vector whose components all equal $n^{*}$.

Define the mappings $g:[1,+\infty) \rightarrow \mathbb{R}_{+}$and $\tilde{s}:[0, X] \times[0, Y] \rightarrow[0,1]$ by

$$
g(n)=n^{1-\alpha \lambda-\beta \sigma-\tau} \cdot a(n)
$$


and

$$
\tilde{s}(x, y)=(Y-y)^{\lambda} \cdot(X-\phi x)^{\sigma} \cdot\left(\rho \gamma x+\frac{\theta y}{k}\right)^{\tau},
$$

respectively. Then note that given that all women choose $n^{*}$, a woman's reproductive success is separable in her time allocation $x$ and the number of children $n^{*}$, since $F$ can then be written:

$$
F\left(n^{*}, x,\left(n^{*}\right)^{(k-1)}, y^{*}\right)=k^{-\alpha \lambda} \cdot g\left(n^{*}\right) \cdot \tilde{s}\left(x, y^{*}\right) .
$$

Likewise, given that all women choose $n^{*}$, the man's reproductive success is also separable in his time allocation $y$ and the number of children per woman $n^{*}$ :

$$
\begin{aligned}
M\left(\boldsymbol{n}^{*}, \boldsymbol{x}^{*}, y\right) & =k \cdot F\left(n^{*}, x,\left(n^{*}\right)^{(k-1)}, y^{*}\right) \\
& =k^{1-\alpha \lambda} \cdot g\left(n^{*}\right) \cdot \tilde{s}\left(x^{*}, y\right) .
\end{aligned}
$$

Since $k^{1-\alpha \lambda} \cdot g\left(n^{*}\right) \geq k^{-\alpha \lambda} \cdot g\left(n^{*}\right)>0$ for any $k \geq 1$ and any $n^{*}$ (where the strict inequality follows from revealed preference) the last two equations in (28) are equivalent to

$$
\left\{\begin{array}{c}
x^{*} \in \arg \max _{x \in[0, X]} \tilde{s}\left(x, y^{*}\right) \\
y^{*} \in \arg \max _{y \in[0, Y]} \tilde{S}\left(x^{*}, y\right),
\end{array}\right.
$$

implying that any equilibrium female-symmetric household time allocation $\left(x^{*}, y^{*}\right) \in[0, X] \times[0, Y]$ is independent of the number of children $n^{*}$ per wife. The next part of the proof characterizes the set of female-symmetric equilibrium household time allocations $\left(x^{*}, y^{*}\right) \in[0, X] \times[0, Y]$.

To begin, note that $y=Y$ cannot be part of an equilibrium strategy profile. Suppose, to the contrary, that $\left(x^{*}, y^{*}\right)=\left(x^{*}, Y\right)$ for some $x^{*} \in[0, X]$. Then $\tilde{s}\left(x^{*}, y^{*}\right)=0$ for any $x^{*} \in[0, X]$, while for any $y \in(0, Y), \tilde{s}\left(x^{*}, y\right)>0$. Similarly, $\left(x^{*}, y^{*}\right)=(0,0)$ cannot be an equilibrium strategy profile. Indeed, $\tilde{s}(0,0)=0$, while, for any $y \in(0, Y), \tilde{s}(0, y)>0$.

Next, it is straightforward to verify that, for each ecology $\omega$ and each $y \in[0, Y), \tilde{s}(x, y)$ is strictly concave in $x$. Likewise, for each female time allocation $x \in[0, X], \tilde{s}$ is strictly concave in $y$. Hence, it is sufficient to study the first-order partial derivatives of $\tilde{s}(x, y)$ to determine the best response functions.

Thus, for the man:

$$
\begin{aligned}
\frac{\partial \tilde{s}(x, y)}{\partial y}= & \frac{\tau \theta}{k} \cdot(Y-y)^{\lambda} \cdot(X-\phi x)^{\sigma} \cdot\left(\rho \gamma x+\frac{\theta y}{k}\right)^{\tau-1} \\
& -\lambda(Y-y)^{\lambda-1}(X-\phi x)^{\sigma} \cdot\left(\rho \gamma x+\frac{\theta y}{k}\right)^{\tau} .
\end{aligned}
$$

Dividing this by the strictly positive term $(Y-y)^{\lambda-1} \cdot(X-\phi x)^{\sigma} \cdot\left(\rho \gamma x+\frac{\theta y}{k}\right)^{\tau-1}$ and simplifying, one 
obtains that of $\frac{\partial \tilde{s}(x, y)}{\partial y}$ has the same sign as

$$
Y \theta \tau-k \lambda \rho \gamma x-\theta(\lambda+\tau) y
$$

This expression being strictly decreasing in $y$, it is non-negative for all $y \in[0, Y]$ iff it is nonnegative for $y=Y$, i.e., if $Y \theta \tau-k \lambda \rho \gamma x-\theta(\lambda+\tau) Y \geq 0$, which is false. The expression in (34) is non-positive for all $y \in[0, Y]$ iff it is non-positive for $y=0$, i.e., iff $Y \theta \tau-k \lambda \rho \gamma x \leq 0$, or

$$
x \geq Y \theta \tau /(k \lambda \rho \gamma) \equiv x_{1}
$$

Thus, if $x \in\left(0, x_{1}\right)$, there exists a unique $y$ such that the expression in (34) equals zero:

$$
y=\frac{Y \tau}{\lambda+\tau}-\frac{k \lambda \rho \gamma}{\theta(\lambda+\tau)} x
$$

and I conclude that the male's best response to the female strategy $x$ (where $x$ is chosen by each of the $k$ women) is:

$$
\left\{\begin{array}{c}
y^{m}=0 \text { if } x \in\left[x_{1}, X\right] \\
y^{m}=\frac{Y \tau}{\lambda+\tau}-\frac{k \lambda \rho \gamma}{\theta(\lambda+\tau)} x \text { if } x \in\left[0, x_{1}\right] .
\end{array}\right.
$$

Turning now to the (representative) woman's best response:

$$
\begin{aligned}
\frac{\partial \tilde{s}(x, y)}{\partial x}= & \tau \rho \gamma \cdot(Y-y)^{\lambda} \cdot(X-\phi x)^{\sigma} \cdot\left(\rho \gamma x+\frac{\theta y}{k}\right)^{\tau-1} \\
& -(Y-y)^{\lambda} \cdot \phi \sigma \cdot(X-\phi x)^{\sigma-1} \cdot\left(\rho \gamma x+\frac{\theta y}{k}\right)^{\tau} .
\end{aligned}
$$

Dividing this by the strictly positive term $(Y-y)^{\lambda} \cdot(X-\phi x)^{\sigma-1} \cdot\left(\rho \gamma x+\frac{\theta y}{k}\right)^{\tau-1}$ and simplifying, one obtains that $\frac{\partial \tilde{s}(x, y)}{\partial x}$ has the same sign as

$$
X k \tau \rho \gamma-(\sigma+\tau) k \rho \gamma \phi x-\sigma \phi \theta y
$$

This expression being strictly decreasing in $x$, it is non-negative for all $x \in[0, X]$ iff it is nonnegative for $x=X$, i.e., iff

$$
y \leq\left[\frac{k \tau \rho \gamma}{\sigma \phi \theta}-\frac{(\sigma+\tau) k \rho \gamma}{\sigma \theta}\right] X \equiv y_{0} .
$$

Note that $y_{0} \geq 0$ iff $\frac{\tau}{\tau+\sigma} \geq \phi$. The expression in (38) is non-positive for all $x \in[0, X]$ iff it is nonpositive for $x=0$, i.e., iff $X k \tau \rho \gamma-\sigma \phi \theta y \leq 0$, or

$$
y \geq X k \tau \rho \gamma /(\sigma \phi \theta) \equiv y_{1} .
$$




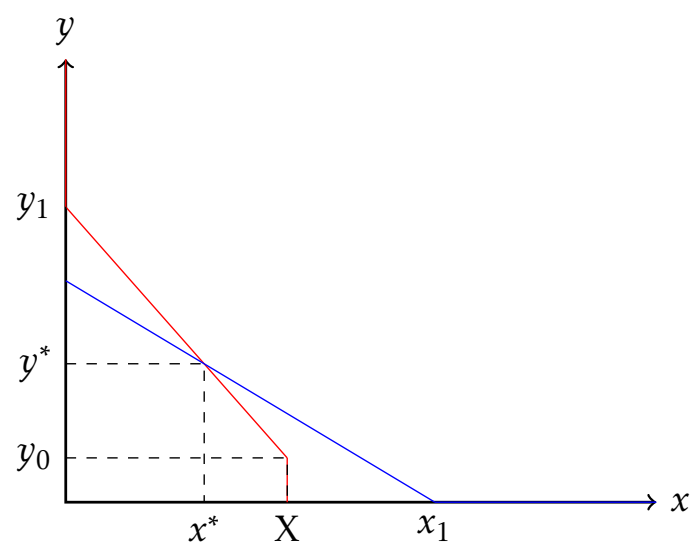

Figure 1: Woman's (red) and Man's (blue) best response curves

Thus, if $y \in\left(\max \left\{0, y_{0}\right\}, y_{1}\right)$, there exists a unique $x$ such that the expression in (38) equals zero:

$$
x=\frac{X \tau}{(\sigma+\tau) \phi}-\frac{\sigma \theta}{(\sigma+\tau) k \rho \gamma} y .
$$

I conclude that a female's best response to the male strategy $y$ is:

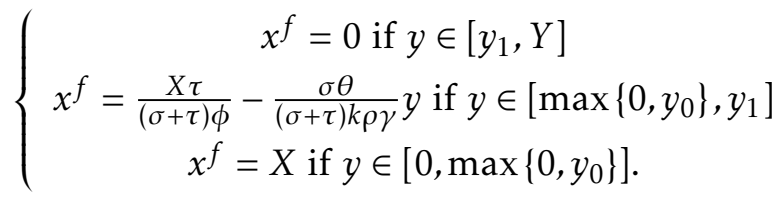

The figure below shows the woman's best response curve in red, and the man's best response curve in blue.

Inspection of this figure indicates that a sufficient condition for there to exist a unique equilibrium is that the (absolute value of the) slope of the man's best response curve (for values of $x \in\left[0, x_{1}\right]$ ) be strictly smaller than the (absolute value of the) inverse of the slope of the (representative) woman's best response curve (for values of $y \in\left[y_{0}, y_{1}\right]$ ), which is true: $\left|\frac{d y^{m}}{d x}\right|=\frac{k \lambda \rho \gamma}{\theta(\lambda+\tau)}<\frac{(\sigma+\tau) k \rho \gamma}{\sigma \theta}=\left|1 /\left(\frac{d x^{f}}{d y}\right)\right|$.

Combining (36) and (40), two cases may be distinguished, depending on whether $y_{0} \leq 0$ or $y_{0}>0$. First, if $\frac{\tau}{\sigma+\tau} \leq \phi$ (i.e., if $y_{0} \leq 0$ ),

$$
\left(x^{*}, y^{*}\right)=\left\{\begin{array}{l}
\left(\frac{X \tau}{\phi(\sigma+\tau)}, 0\right) \text { if } \frac{\theta}{\rho \gamma} \leq \frac{k \lambda}{\phi(\sigma+\tau)} \cdot \frac{X}{Y} \\
\left(\frac{X(\lambda+\tau) \rho \gamma k-Y \sigma \phi \theta}{\rho \gamma k \phi(\lambda+\sigma+\tau)}, \frac{Y \theta \phi(\sigma+\tau)-X \rho \gamma \lambda k}{\theta \phi(\lambda+\sigma+\tau)}\right) \text { if } \frac{\theta}{\rho \gamma} \in\left[\frac{k \lambda}{\phi(\sigma+\tau)} \cdot \frac{X}{Y}, \frac{k(\lambda+\tau)}{\sigma \phi} \cdot \frac{X}{Y}\right] \\
\left(0, \frac{Y \tau}{\lambda+\tau}\right) \text { if } \frac{\theta}{\rho \gamma} \geq \frac{k(\lambda+\tau)}{\sigma \phi} \cdot \frac{X}{Y} .
\end{array}\right.
$$


Second, if $\frac{\tau}{\sigma+\tau}>\phi$ (i.e., if $y_{0}>0$ )

$$
\left(x^{*}, y^{*}\right)=\left\{\begin{array}{l}
(X, 0) \text { if } \frac{\theta}{\rho \gamma} \leq \frac{k \lambda}{\tau} \cdot \frac{X}{Y} \\
\left(X, \frac{Y \tau \theta-X k \lambda \rho \gamma}{\theta(\lambda+\tau)}\right) \text { if } \frac{\theta}{\rho \gamma} \in\left[\frac{k \lambda}{\tau} \cdot \frac{X}{Y}, \frac{k[\lambda+\tau-\phi(\lambda+\sigma+\tau)]}{\sigma \phi} \cdot \frac{X}{Y}\right] \\
\left(\frac{X(\lambda+\tau) \rho \gamma k-Y \sigma \phi \theta}{\rho \gamma k \phi(\lambda+\sigma+\tau)}, \frac{Y \theta \phi(\sigma+\tau)-X \rho \gamma \lambda k}{\theta \phi(\lambda+\sigma+\tau)}\right) \text { if } \frac{\theta}{\rho \gamma} \in\left[\frac{k[\lambda+\tau-\phi(\lambda+\sigma+\tau)]}{\sigma \phi} \cdot \frac{X}{Y}, \frac{k(\lambda+\tau)}{\sigma \phi} \cdot \frac{X}{Y}\right] \\
\left(0, \frac{Y \tau}{\lambda+\tau}\right) \text { if } \frac{\theta}{\rho \gamma} \geq \frac{k(\lambda+\tau)}{\sigma \phi} \cdot \frac{X}{Y} .
\end{array}\right.
$$

The last part of the proof concerns the equilibrium number of children per woman. To begin, note that if there is only one wife $(k=1)$ she chooses the number of children $n$ that maximizes

$$
n^{1-\alpha \lambda-\beta \sigma-\tau} \cdot a(n) \cdot\left(Y-y^{*}\right)^{\lambda} \cdot\left(X-\phi x^{*}\right)^{\sigma} \cdot\left(\rho \gamma x^{*}+\theta y^{*}\right)^{\tau}
$$

or $g(n) \cdot \tilde{s}\left(x^{*}, y^{*}\right)$, where $g(n)$ was defined in (29). Since $\left(x^{*}, y^{*}\right)$ does not depend on $n$, this amounts to choosing $n$ to maximize $g(n)$. If interior $(n>1)$, the solution must satisfy the first-order condition

$$
g^{\prime}(n)=(1-\lambda \alpha-\sigma \beta-\tau)(1-b n) \cdot n^{-\lambda \alpha-\sigma \beta-\tau}-b n^{1-\lambda \alpha-\sigma \beta-\tau}=0
$$

Hence, two cases arise.

Case A: $1-\lambda \alpha-\sigma \beta-\tau \leq 0$. Then $g^{\prime}(n)<0$ for all $n \in[1,+\infty)$, in which case $n^{*}=1$.

Case B: $1-\lambda \alpha-\sigma \beta-\tau>0$. Then the second derivative

$$
g^{\prime \prime}(n)=(1-\lambda \alpha-\sigma \beta-\tau)\left[-(\lambda \alpha+\sigma \beta+\tau)(1-b n) n^{-\lambda \alpha-\sigma \beta-\tau-1}-2 b n^{-\lambda \alpha-\sigma \beta-\tau}\right]
$$

is strictly negative, so that either $n^{*}=1$ (if $g^{\prime}(1) \leq 0$ ), or there exists $n>1$ that satisfies the necessary first-order condition for an interior solution, $g^{\prime}(n)=0$. Since $g^{\prime}(n)=0$ iff $n=\frac{1-\lambda \alpha-\sigma \beta-\tau}{(2-\lambda \alpha-\sigma \beta-\tau) b}$, the solution for $k=1$ writes:

$$
n^{*}(1)=\max \left\{1, \frac{1-\lambda \alpha-\sigma \beta-\tau}{(2-\lambda \alpha-\sigma \beta-\tau) b}\right\} .
$$

Turning now to the general case $k>1$, an interior equilibrium $n^{*}>1$ must satisfy the first-order condition:

$$
\left[g^{\prime}(n) \cdot s\left(n, x^{*},\left(n^{*}\right)^{(k-1)}, y^{*}\right)+g(n) \cdot s_{1}\left(n, x^{*},\left(n^{*}\right)^{(k-1)}, y^{*}\right)\right]_{\mid n=n^{*}}=0,
$$

where $s_{1}$ denotes the partial derivative of $s$ with respect to its first argument. Since $s(\cdot)>0$, $g(n)>0$, and $s_{1}(\cdot)<0$, this equation implies that at an interior equilibrium $n^{*}, g^{\prime}\left(n^{*}\right)>0$, which in turn implies that for any $k$ for which $n^{*}(k)>0, n^{*}(k)<n^{*}(1)$. Note that this further means that if $1-\lambda \alpha-\sigma \beta-\tau \leq 0$, then $n^{*}(k)=1$ for all $k \geq 1$. Finally, note that since the absolute value of $s_{1}$ increases while $s$ decreases as $k$ increases (ceteris paribus), this equation further implies that $n^{*}(k)$ is strictly decreasing in $k$. 


\subsection{Proof of Proposition 3}

The proof is in two steps. I begin by proving that even if a man could impose his most preferred female-symmetric child production allocation on his wives, his reproductive success would not necessarily be increasing in the number of wives. I will then show that this result, together with Proposition 1, implies that his reproductive success would not necessarily be increasing in the number of wives under the assumption that women do have agency over their fertility and time allocation decisions. This modeling strategy simplifies the calculations (note that it also delivers one robustness check as a by-product).

Step 1: Analysis for a (hypothetical) man who would be able to choose his preferred femalesymmetric child production allocation.

Consider a man who has $k$ wives, and who chooses $n$ and $(x, y)$ to maximize

$$
\widehat{M}(n, x, y)=k \cdot n \cdot a(n) \cdot\left[\frac{Y-y}{(k n)^{\alpha}}\right]^{\lambda} \cdot\left(\frac{X-\phi x}{n^{\beta}}\right)^{\sigma} \cdot\left(\frac{\rho \gamma x}{n}+\frac{\theta y}{k n}\right)^{\tau} .
$$

Then:

Lemma 2. For any ecology $\omega$, any number of wives $k \geq 1$, and any amount of labor resources $Y \geq 0$, there exists a unique female-symmetric child production allocation $(\widehat{n}, \widehat{x}, \widehat{y})$ that maximizes male reproductive success $\widehat{M}(x, y, n)$. Furthermore, $\widehat{x}=x^{*}$ and $\widehat{y}=y^{*}$.

Proof: To begin, note that the man's maximization problem is separable in $n$ and $(x, y)$, since the objective function in (48) can be written

$$
\widehat{M}(n, x, y)=k^{1-\alpha \lambda} \cdot g(n) \cdot \tilde{s}(x, y),
$$

where $g(n)$ was defined in (29) and $\tilde{s}(x, y)$ in (30). Specifically, choosing $(n, x, y) \in[1,+\infty) \times[0, X] \times$ $[0, Y]$ to maximize $\widehat{M}(n, x, y)$ boils down to choosing $n \in[1,+\infty)$ to maximize $g(n)$ and $(x, y) \in$ $[0, X] \times[0, Y]$ to maximize $\tilde{s}(x, y)$. In view of the system of equations $(32)$, which defines the unique Nash equilibrium time allocations $x^{*}$ and $y^{*}$ in game $\Gamma$, this observation clearly implies that the solution entails setting $x=x^{*}$ and $y=y^{*}$.

Turning now to the choice of $n$, it is immediate from the end of the proof of Proposition 1 (see (46)) that

$$
\widehat{n}=\max \left\{1, \frac{1-\lambda \alpha-\sigma \beta-\tau}{(2-\lambda \alpha-\sigma \beta-\tau) b}\right\}
$$

\section{Q.E.D.}

Writing the man's preferred female-symmetric fertility and time allocations $\widehat{n}, \widehat{x}$, and $\widehat{y}$ as functions of the number of wives $k \geq 1$ and the man's time budget $Y$, the reproductive success 
that the man achieves can be written as a function of $k$ and $Y$ :

$$
\widetilde{M}(k, Y) \equiv \widehat{M}(\widehat{n}(k, Y), \widehat{x}(k, Y), \widehat{y}(k, Y)) .
$$

The following lemma shows how $\widetilde{M}$ varies with $k$, holding $Y$ constant. In this lemma,

$$
\hat{\alpha} \equiv \frac{\rho \gamma x^{*}(1, Y)+(1-\tau) \theta y^{*}(1, Y)}{\lambda\left[\rho \gamma x^{*}(1, Y)+\theta y^{*}(1, Y)\right]}
$$

and (for $\alpha \lambda<1-\tau$ ) the threshold value $\hat{k}$ is implictly defined by the equation

$$
(1-\lambda \alpha) \cdot \rho \gamma x^{*}(\hat{k}, Y)=(\tau-1+\lambda \alpha) \cdot \frac{\theta y^{*}(\hat{k}, Y)}{\hat{k}}
$$

Lemma 3. Consider a man who, for any given number of wives $k \geq 1$ and labor resources $Y$, can choose $(n, x, y)$ so as to achieve reproductive success $\widetilde{M}(k, Y)$ (see (50)). For such a man:

(i) if $y^{*}(1, Y)=0$ or if $\alpha \leq \hat{\alpha}$, then $\frac{\partial \widetilde{M}(k, Y)}{\partial k} \geq 0$ for all $k \geq 1$ (the inequality being strict if $y^{*}(1, k)=0$ and $\alpha \lambda<1$, or if $\alpha<\hat{\alpha}$ );

(ii) if $y^{*}(1, k)>0$ and $\alpha>\hat{\alpha}$, then there exists $\hat{k}>1$ such that $\frac{\partial \widetilde{M}(k, Y)}{\partial k}<0$ for all $k \in(1, \hat{k})$ and $\frac{\partial \widetilde{M}(k, Y)}{\partial k}>0$ for all $k>\hat{k}$;

(iii) if $\alpha=\lambda=1$, then $\widetilde{M}(1, Y) \geq \widetilde{M}(k, Y)$ for all $k>1$, the inequality being strict if and only if $y^{*}(1, Y)>0$.

Proof: Using the notation introduced in the proof of Lemma 2, and letting

$$
s^{*}\left(x^{*}(k, Y), y^{*}(k, Y), k\right)=\left[Y-y^{*}(k, Y)\right]^{\lambda} \cdot\left[X-\phi x^{*}(k, Y)\right]^{\sigma} \cdot\left[\rho \gamma x^{*}(k, Y)+\frac{\theta y^{*}(k, Y)}{k}\right]^{\tau} \text {, }
$$

one obtains the following expression for male reproductive success as a function of $k$ (see equation $(50))$ :

$$
\begin{aligned}
\widetilde{M}(k, Y) & =M\left(x^{*}(k, Y), y^{*}(k, Y), \widehat{n}, k\right) \\
& =g(\widehat{n}) \cdot k^{1-\lambda \alpha} \cdot s^{*}\left(x^{*}(k, Y), y^{*}(k, Y), k\right),
\end{aligned}
$$

where $\widehat{n}$ is defined in (46). Upon dividing the expression in (54) by the strictly positive constant $g(\widehat{n})$, one obtains that $\frac{\partial \widetilde{M}(k, Y)}{\partial k}$ has the same sign as

$$
\begin{aligned}
& (1-\lambda \alpha) \cdot k^{-\lambda \alpha} \cdot s^{*}\left(x^{*}(k, Y), y^{*}(k, Y), k\right) \\
& +k^{1-\lambda \alpha} \cdot \frac{d s^{*}\left(x^{*}(k, Y), y^{*}(k, Y), k\right)}{d k}
\end{aligned}
$$


where

$$
\begin{aligned}
\frac{d s^{*}\left(x^{*}(k, Y), y^{*}(k, Y), k\right)}{d k}= & \left.\frac{\partial \tilde{s}(x, y)}{\partial x}\right|_{(x, y)=\left(x^{*}(k, Y), y^{*}(k, Y)\right)} \cdot \frac{\partial x^{*}(k, Y)}{\partial k} \\
& +\left.\frac{\partial \tilde{s}(x, y)}{\partial y}\right|_{(x, y)=\left(x^{*}(k, Y), y^{*}(k, Y)\right)} \cdot \frac{\partial y^{*}(k, Y)}{\partial k} \\
& +\frac{\partial s^{*}\left(x^{*}(k, Y), y^{*}(k, Y), k\right)}{\partial k}
\end{aligned}
$$

(for the definition of $\tilde{s}(x, y)$, see (30)). From the proof of Proposition 1, one obtains

$$
\left.\frac{\partial \tilde{s}(x, y)}{\partial x}\right|_{(x, y)=\left(x^{*}(k, Y), y^{*}(k, Y)\right)} \cdot \frac{\partial x^{*}(k, Y)}{\partial k}=\left.\frac{\partial \tilde{s}(x, y)}{\partial y}\right|_{(x, y)=\left(x^{*}(k, Y), y^{*}(k, Y)\right)} \cdot \frac{\partial y^{*}(k, Y)}{\partial k}=0,
$$

so that (56) reduces to

$$
\begin{gathered}
\frac{d s^{*}\left(x^{*}(k, Y), y^{*}(k, Y), k\right)}{d k}=\frac{\partial s^{*}\left(x^{*}(k, Y), y^{*}(k, Y), k\right)}{\partial k} \\
=-\frac{\tau \theta y^{*}(k, Y)}{k^{2}} \cdot\left[Y-y^{*}(k, Y)\right]^{\lambda} \cdot\left[X-\phi x^{*}(k, Y)\right]^{\sigma} \cdot\left[\rho \gamma x^{*}(k, Y)+\frac{\theta y^{*}(k, Y)}{k}\right]^{\tau-1} \\
=-\frac{\tau \theta y^{*}(k, Y)}{k^{2}} \cdot\left[\rho \gamma x^{*}(k, Y)+\frac{\theta y^{*}(k, Y)}{k}\right]^{-1} \cdot s^{*}\left(x^{*}(k, Y), y^{*}(k, Y), k\right) .
\end{gathered}
$$

Plugging this into (55), dividing by $s^{*}\left(x^{*}(k, Y), y^{*}(k, Y), k\right) \cdot k^{-\lambda \alpha} \cdot\left[\rho \gamma x^{*}(k, Y)+\frac{\theta y^{*}(k, Y)}{k}\right]^{-1}$ (which is strictly positive), and rearranging the terms, one obtains that $\frac{\partial \widetilde{M}(k, Y)}{\partial k}$ has the same sign as

$$
A(k) \equiv(1-\lambda \alpha) \cdot\left[\rho \gamma x^{*}(k, Y)+\frac{\theta y^{*}(k, Y)}{k}\right]-\tau \cdot \frac{\theta y^{*}(k, Y)}{k} .
$$

Recalling that $y^{*}(k, Y)$ is decreasing in $k$, let $\tilde{k} \geq 0$ denote the threshold value such that $y^{*}(k, Y)>0$ iff $k<\tilde{k}$, and $y^{*}(k, Y)=0$ iff $k \geq \tilde{k}$ (note that $\tilde{k}$ may be smaller than 1 ):

$$
\tilde{k} \equiv\left\{\begin{array}{l}
\frac{\theta \phi(\sigma+\tau)}{\rho \gamma \lambda} \cdot \frac{Y}{X} \text { if } \frac{\tau}{\sigma+\tau} \leq \phi \\
\frac{\theta \tau}{\rho \gamma \lambda} \cdot \frac{Y}{X} \text { if } \frac{\tau}{\sigma+\tau}>\phi
\end{array}\right.
$$

Now note the following:

Remark 1. $\frac{y^{*}(k, Y)}{k}$ is strictly decreasing in $k$ for any $k<\tilde{k}$ and constant in $k$ for any $k \geq \tilde{k}$.

Remark 2. For any $(\alpha, \lambda) \in[0,1] \times(0,1], 1-\lambda \alpha \geq 0$. Furthermore, $1-\lambda \alpha=0$ if and only if $\alpha=\lambda=1$, in which case $A(k)$ has the same sign as $-y^{*}(k, Y)$. The remaining remarks pertain to the case $\alpha \cdot \lambda<1$.

Remark 3. The term in square brackets in (58) (which is the total amount of food produced for 
each brood of $\widehat{n}$ children) writes

$$
\rho \gamma x^{*}(k, Y)+\frac{\theta y^{*}(k, Y)}{k}=\left\{\begin{array}{l}
\frac{\tau \rho \gamma X}{\phi(\sigma+\tau)} \text { if } k \geq \frac{\theta \phi(\sigma+\tau)}{\rho \gamma \lambda} \cdot \frac{Y}{X} \\
\frac{\tau(\rho \gamma k X+\theta \phi Y)}{k \phi(\lambda+\sigma+\tau)} \text { if } k \in\left[\frac{\theta \phi \sigma}{\rho \gamma(\lambda+\tau)} \cdot \frac{Y}{X}, \frac{\theta \phi(\sigma+\tau)}{\rho \gamma \lambda} \cdot \frac{Y}{X}\right] \\
\frac{\tau \theta Y}{k(\lambda+\tau)} \text { if } k \leq \frac{\theta \phi \sigma}{\rho \gamma(\lambda+\tau)} \cdot \frac{Y}{X}
\end{array}\right.
$$

if $\frac{\tau}{\sigma+\tau} \leq \phi$, and

$$
\rho \gamma x^{*}(k, Y)+\frac{\theta y^{*}(k, Y)}{k}=\left\{\begin{array}{l}
\rho \gamma X \text { if } k \geq \frac{\theta \tau}{\rho \gamma \lambda} \cdot \frac{Y}{X} \\
\frac{\tau(\rho \gamma k X+\theta Y)}{k(\lambda+\tau)} \text { if } k \in\left[\frac{\theta \phi \sigma}{\rho \gamma[\lambda+\tau-\phi(\lambda+\sigma+\tau)]} \cdot \frac{Y}{X}, \frac{\theta \tau}{\rho \gamma \lambda} \cdot \frac{Y}{X}\right] \\
\frac{\tau(\rho \gamma k X+\theta \phi Y)}{k \phi(\lambda+\sigma+\tau)} \text { if } k \in\left[\frac{\theta \phi \sigma}{\rho \gamma(\lambda+\tau)} \cdot \frac{Y}{X}, \frac{\theta \phi \sigma}{\rho \gamma[\lambda+\tau-\phi(\lambda+\sigma+\tau)]} \cdot \frac{Y}{X}\right] \\
\frac{\tau \theta Y}{k(\lambda+\tau)} \text { if } k \leq \frac{\theta \phi \sigma}{\rho \gamma(\lambda+\tau)} \cdot \frac{Y}{X}
\end{array}\right.
$$

if $\frac{\tau}{\sigma+\tau}>\phi$. In either case, this term is always strictly positive. Furthermore, it is strictly decreasing in $k$ for any $k<\tilde{k}$ and constant in $k$ for any $k \geq \tilde{k}$.

Remark 4. Suppose that $\tilde{k}>1$. Then $A(k)$ changes sign at most once for $k \in[1, \tilde{k})$. Indeed, suppose that there exists some $\hat{k} \in[1, \tilde{k})$ such that $A(\hat{k})=0$, i.e.,

$$
(1-\lambda \alpha) \cdot \rho \gamma x^{*}(\hat{k}, Y)=(\tau-1+\lambda \alpha) \cdot \frac{\theta y^{*}(\hat{k}, Y)}{\hat{k}} .
$$

(Note that $\hat{k}$ must indeed be strictly smaller that $\tilde{k}$ since $A(k)>0$.for any $k \geq \tilde{k}$.) Since $\rho \gamma x^{*}(k, Y)+\frac{\theta y^{*}(k, Y)}{k}>0$ for all $k$, and since $(1-\lambda \alpha) \cdot \rho \gamma>0$, both the left-hand side and the right-hand side of this expression must be strictly positive. Since $x^{*}(k, Y)$ is increasing in $k$ and $\frac{\theta y^{*}(\hat{k}, Y)}{\hat{k}}$ is decreasing in $k$, it must be that $(1-\lambda \alpha) \cdot \rho \gamma x^{*}(k, Y)>(\tau-1+\lambda \alpha) \cdot \frac{\theta y^{*}(k, Y)}{k}$ for any $k>\hat{k}$.

Remarks 1 - 4 together imply:

A. If $\alpha=\lambda=1$ and $y^{*}(1)=0$, then $\frac{\partial \widetilde{M}(k, Y)}{\partial k}=0$ for all $k \geq 1$.

B. If $\alpha=\lambda=1$ and $y^{*}(1)>0$, then $\frac{\partial \widetilde{M}(k, Y)}{\partial k}<0$ for all $k \in[1, \tilde{k})$ and $\frac{\partial \widetilde{M}(k, Y)}{\partial k}=0$ for all $k \geq \tilde{k}$.

C. If $\alpha \lambda<1$ and $y^{*}(1)=0$, then $\frac{\partial \widetilde{M}(k, Y)}{\partial k}>0$ for all $k \geq 1$.

D. If $\alpha \lambda<1$ and $y^{*}(1)>0$, then $\frac{\partial \widetilde{M}(k, Y)}{\partial k}>0$ for all $k \geq \tilde{k}>1$. Moreover, a sufficient condition for $\widetilde{M}$ to be non-monotonic in $k$ is that $\left.\frac{\partial \widetilde{M}(k, Y)}{\partial k}\right|_{k=1}<0$, which is true if and only if

$$
(1-\lambda \alpha)\left[\rho \gamma x^{*}(1, Y)+\theta y^{*}(1, Y)\right]<\tau \theta y^{*}(1, Y)
$$


Because $x^{*}(1, Y)$ and $y^{*}(1, Y)$ do not depend on $\alpha$, and since $\rho \gamma x^{*}(1, Y)+\theta y^{*}(1, Y)>0$, the left-hand side can be viewed as an affine and strictly decreasing function of $\alpha$, which takes the value 0 for $\alpha=1 / \lambda$ and the value $\rho \gamma x^{*}(1, Y)+\theta y^{*}(1, Y)>0$ for $\alpha=0$. Hence, (63) is equivalent to

$$
\alpha>\frac{\rho \gamma x^{*}(1, Y)+(1-\tau) \theta y^{*}(1, Y)}{\lambda\left[\rho \gamma x^{*}(1, Y)+\theta y^{*}(1, Y)\right]} \equiv \hat{\alpha}
$$

Because $A(k)$ changes sign at most once (see Remark 4), the condition $\alpha>\hat{\alpha}$ is also necessary for $\widetilde{M}$ to be non-monotonic in $k$. Furthermore, if $\alpha>\hat{\alpha}$, there exists $\hat{k} \in(1, \tilde{k})$ such that $\frac{\partial \widetilde{M}(k, Y)}{\partial k}<0$ for all $k \in[1, \hat{k}), \frac{\partial \widetilde{M}(k, Y)}{\partial k}<0$ if $k=\hat{k}$, and $\frac{\partial \widetilde{M}(k, Y)}{\partial k}>0$ for all $k>\hat{k}$. Finally, if $\alpha \leq \hat{\alpha}$, $\frac{\partial \widetilde{M}(k, Y)}{\partial k} \geq 0$ for all $k \geq 1$, with a strict inequality for all $k \geq 1$ if and only if $\alpha<\hat{\alpha}$.

\section{Q.E.D.}

\section{Step 2: Analysis of the implications of the analysis in Step 1 for the model in the text.}

Returning to the case where the unique Nash equilibrium of the non-cooperative game $\Gamma$ is played in each household, consider the equilibrium male reproductive success $M^{*}(k, y)$, which can be written:

$$
M^{*}(k, Y)=g\left(n^{*}(k)\right) \cdot k^{1-\lambda \alpha} \cdot s^{*}\left(x^{*}(k, Y), y^{*}(k, Y), k\right),
$$

where $n^{*}(k)$ was defined in (46) and (47), and $s^{*}(\cdot)$ in (53). Using the expression for the reproductive success that a male who can impose his preferred female-symmetric allocation on his wives (see (54)), I obtain

$$
M^{*}(k, Y)=\frac{g\left(n^{*}(k)\right)}{g(\widehat{n})} \cdot \widetilde{M}(k, Y) .
$$

Referring to the same cases as in the proof of Proposition 1, I immediately obtain:

Case A: $1-\lambda \alpha-\sigma \beta-\tau \leq 0$. Then $n^{*}(k)=\widehat{n}=1$ for all $k \geq 1$, so that $\frac{g\left(n^{*}(k)\right)}{g(\widehat{n})}=1$ and $M^{*}(k, Y)=$ $\widetilde{M}(k, Y)$ for all $k \geq 1$.

Case B: $1-\lambda \alpha-\sigma \beta-\tau>0$. Then, for all $k>1, n^{*}(k)<\widehat{n}=n^{*}(1)$, and hence $M^{*}(1, Y)=\widetilde{M}(1, Y)$ while $M^{*}(k, Y)<\widetilde{M}(k, Y)$ for all $k>1$.

The statement in Proposition 3 follows from these results together with Lemma 3. To see this, note that:

1. there exist ecologies $\omega$ such that (a) $y *(1, Y)=0$, (b) $n^{*}(k)=\widehat{n}$ for all $k \geq 1$, and (c) $\frac{\partial \widetilde{M}(k, Y)}{\partial k}>0$ for all $k \geq 1$; for example $1-\lambda \alpha-\sigma \beta-\tau \leq 0$ and $\frac{\theta}{\rho \gamma} \leq \frac{\lambda}{\phi(\sigma+\tau)} \cdot \frac{X}{Y}$; this proves that $\Omega_{1} \neq \emptyset$;

2. in ecologies such that $\frac{\partial \widetilde{M}(k, Y)}{\partial k}<0$ for small values of $k$ : since $M^{*}(1, Y)=\widetilde{M}(1, Y)$ and $M^{*}(k, Y) \leq$ $\widetilde{M}(k, Y)$ for all $k>1$, and both $M^{*}$ and $\widetilde{M}$ are continuous in $k, M^{*}$ is strictly decreasing in $k$ for small enough values of $k$; this proves that $\Omega_{2} \neq \emptyset$;

3. there exists ecologies such that $\frac{\partial \widetilde{M}(k, Y)}{\partial k}<0$ and $n^{*}(k)=\widehat{n}$ for all $k \geq 1$; the above arguments then imply that $M^{*}(k, Y)=\widetilde{M}(k, Y)$ for all $k \geq 1$; this proves that $\Omega_{3} \neq \emptyset$. 


\subsection{Proof of Proposition 5}

The results in the proposition follow from standard analysis (see Weibull, 1995, p.40), by noting that since $\frac{1}{2} M^{*}(2, Y)>0$ the game is:

1. a Coordination Game if $M^{*}(1, Y)>M^{*}(2, Y)$; then both pure strategies are evolutionarily stable;

2. a Prisoner's Dilemma if $M^{*}(2, Y)>M^{*}(1, Y)$ : then only the "non-cooperative strategy" $r=1$ is evolutionarily stable;

3. never a Hawk-Dove game: this explains why there is no ecology for which there exists an evolutionarily stable mixed strategy.

\subsection{Proof of Proposition 6}

The results in the proposition follow from standard analysis (see Weibull, 1995, p.40). First, for any $\varphi \in(0,2 Y], \frac{1}{2} M^{*}(2, \varphi)>0$ and hence the game is:

1. a Coordination Game if $M^{*}(1, Y)>M^{*}(2, \varphi)$, i.e., if either the ecology $\omega$ is such that $M^{*}(1, Y)>$ $M^{*}(2, Y)$ and $\varphi<Y+A$, where $A>0$ is the amount of male resources such that $M^{*}(1, Y)=$ $M^{*}(2, Y+A)$, or the ecology $\omega$ is such that $M^{*}(1, Y)<M^{*}(2, Y)$ and $\varphi<Y-B$, where $B>0$ is the amount of male resources such that $M^{*}(1, Y)=M^{*}(2, Y-B)$; then both pure strategies are evolutionarily stable;

2. a Prisoner's Dilemma if $M^{*}(2, \varphi)>M^{*}(1, Y)$ : then only the "non-cooperative strategy" $r=1$ is evolutionarily stable;

3. never a Hawk-Dove game: this explains why there is no ecology for which there exists an evolutionarily stable mixed strategy.

Second, consider the case $\varphi=0$. Then $M^{*}(2, \varphi)=0$ and the argument in the text (see (17)) shows that Compete is not evolutionarily stable. I now show that Surrender is evolutionarily stable. Suppose that Surrender (i.e., the mixed strategy $\zeta=0$ ) is the resident strategy and that a share $\varepsilon>0$ of the population carries the mutant strategy $\zeta^{\prime} \neq 0$. The average reproductive success of a resident (who settles down with his teenage girlfriend when matched with another resident and loses with certainty when matched with a mutant who Competes) is then

$$
(1-\varepsilon) \cdot M^{*}(1, Y)+\varepsilon \cdot\left[\zeta^{\prime} \cdot 0+\left(1-\zeta^{\prime}\right) \cdot M^{*}(1, Y)\right],
$$


while that of a mutant (who wins with certainty against a resident when he plays Compete and with probability $1 / 2$ when matched with another mutant who plays Compete) is

$$
\begin{aligned}
& \zeta^{\prime} \cdot\left[\left[1-\varepsilon+\varepsilon\left(1-\zeta^{\prime}\right)\right] \cdot M^{*}(2, \varphi)+\varepsilon \zeta^{\prime} \cdot \frac{M^{*}(2, \varphi)}{2}\right] \\
& +\left(1-\zeta^{\prime}\right) \cdot\left[\left(1-\varepsilon+\varepsilon\left(1-\zeta^{\prime}\right)\right) \cdot M^{*}(1, Y)+\varepsilon \zeta^{\prime} \cdot 0\right] .
\end{aligned}
$$

Strategy $\zeta=0$ is evolutionarily stable against $\zeta^{\prime}=1$ iff there exists some $\bar{\varepsilon}>0$ such that for all $\varepsilon \in(0, \bar{\varepsilon})$ the expression in (67) is strictly larger than the expression in (68). Clearly, by virtue of the continuity of the expressions in $\varepsilon$, a sufficient condition is that when evaluated at $\varepsilon=0$ the expression in (67) is strictly larger than that in (68), a condition which reduces to $M^{*}(1, Y)>$ $M^{*}(2, \varphi)$, which holds for $\varphi=0$ since $M^{*}(2,0)=0$.

Finally, I show that for $\varphi=0$ there exists no mixed strategy $\zeta \in(0,1)$ which is evolutionarily stable. Suppose, to the contrary, that there exists some mixed strategy $\zeta \in(0,1)$ which is evolutionarily stable. Then $\zeta$ must be such that the two pure strategies Compete and Surrender yield the same reproductive success, i.e.:

$$
(1-\zeta) \cdot M^{*}(1, Y)+\zeta \cdot 0=(1-\zeta) \cdot M^{*}(2, \varphi)+\zeta \cdot \frac{M^{*}(2, \varphi)}{2}
$$

However, this equality is violated since $\varphi=0$ implies that the right-hand side is nil.

\subsection{Proof of Proposition 7}

Claim (i): $\zeta=0$ is evolutionarily stable if and only if the ecology $\omega$ is such that $M^{*}(1, Y)>$ $M^{*}(2, Y)$ and $\varphi-Y$ is not too large. Suppose that $\zeta=0$ is the resident strategy and that a share $\varepsilon>0$ of the population carries the mutant strategy $\zeta^{\prime} \neq 0$. The average reproductive success of a resident (who settles down with his teenage girlfriend when matched with another resident and loses with certainty when matched with a mutant who Competes) is then

$$
\operatorname{Pr}[0 \mid 0, \varepsilon] \cdot M^{*}(1, Y)+\operatorname{Pr}\left[\zeta^{\prime} \mid 0, \varepsilon\right] \cdot\left[\zeta^{\prime} \cdot 0+\left(1-\zeta^{\prime}\right) \cdot M^{*}(1, Y)\right]
$$

while that of a mutant (who wins with certainty against a resident when he plays Compete and with probability $1 / 2$ when matched with another mutant who plays Compete) is

$$
\begin{aligned}
& \zeta^{\prime} \cdot\left[\left[\operatorname{Pr}\left[0 \mid \zeta^{\prime}, \varepsilon\right]+\operatorname{Pr}\left[\zeta^{\prime} \mid \zeta^{\prime}, \varepsilon\right]\left(1-\zeta^{\prime}\right)\right] \cdot M^{*}(2, \varphi)+\operatorname{Pr}\left[\zeta^{\prime} \mid \zeta^{\prime}, \varepsilon\right] \cdot \zeta^{\prime} \cdot \frac{M^{*}(2, \varphi)}{2}\right] \\
& +\left(1-\zeta^{\prime}\right) \cdot\left[\left(\operatorname{Pr}\left[0 \mid \zeta^{\prime}, \varepsilon\right]+\operatorname{Pr}\left[\zeta^{\prime} \mid \zeta^{\prime}, \varepsilon\right]\left(1-\zeta^{\prime}\right)\right) \cdot M^{*}(1, Y)+\operatorname{Pr}\left[\zeta^{\prime} \mid \zeta^{\prime}, \varepsilon\right] \zeta^{\prime} \cdot 0\right]
\end{aligned}
$$

Strategy $\zeta=0$ is evolutionarily stable against $\zeta^{\prime} \in(0,1]$ if and only if there exists some $\bar{\varepsilon}>0$ such that for all $\varepsilon \in(0, \bar{\varepsilon})$ the expression in $(70)$ is strictly larger than the expression in (71). Clearly, 
by virtue of the continuity of the expressions in $\varepsilon$ (recall that the conditional probabilities are continuous in $\varepsilon$ ), a sufficient condition is that when evaluated at $\varepsilon=0$ the expression in (70) is strictly larger than that in (71), a condition which reduces to

$$
M^{*}(1, Y)>M^{*}(2, \varphi)-\left[\left(1-\zeta^{\prime}\right) \sigma \cdot M^{*}(1, Y)+\sigma \zeta^{\prime} / 2 \cdot M^{*}(2, \varphi)\right]
$$

where $\sigma=\lim _{\varepsilon \rightarrow 0} \operatorname{Pr}\left[\zeta^{\prime} \mid \zeta^{\prime}, \varepsilon\right]$. Note now that for any $\sigma \in(0,1]$ and any $\zeta^{\prime} \in(0,1]$ the term inside the square brackets in inequality (72) is strictly positive. Recalling the definitions of $A$ and $B$ in the proof of Proposition 6, I can thus conclude that, for any $\zeta^{\prime} \in(0,1]$ :

- if the ecology $\omega$ is such that $M^{*}(1, Y)>M^{*}(2, Y)$, then there exists some $A^{\prime}\left(\zeta^{\prime}\right)>A>0$ such that strategy $\zeta=0$ is evolutionarily stable against $\zeta^{\prime}$ if $\varphi<Y+A^{\prime}\left(\zeta^{\prime}\right)$;

- if the ecology $\omega$ is such that $M^{*}(1, Y)<M^{*}(2, Y)$, then there exists some $B^{\prime}\left(\zeta^{\prime}\right)$, where $B>B^{\prime}\left(\zeta^{\prime}\right)>$ 0 , such that strategy $\zeta=0$ is evolutionarily stable against $\zeta^{\prime}$ if $\varphi<Y-B^{\prime}\left(\zeta^{\prime}\right)$.

Claim (ii): there exists no mixed strategy $\zeta \in(0,1)$ which is evolutionarily stable. Suppose, to the contrary, that there exists some mixed strategy $\zeta \in(0,1)$ which is evolutionarily stable. Then, $\zeta$ must be such that the two pure strategies Compete and Surrender yield the same reproductive success, i.e.:

$$
(1-\zeta) \cdot M^{*}(1, Y)+\zeta \cdot 0=(1-\zeta) \cdot M^{*}(2, \varphi)+\zeta \cdot \frac{M^{*}(2, \varphi)}{2}
$$

Assume now that $\zeta$ is the resident strategy, and consider the mutant strategy $\zeta^{\prime}=0$, represented in a share $\varepsilon$ of the population. Then, residents get, on average, reproductive success equal to

$$
\begin{aligned}
& (1-\zeta)\left[\operatorname{Pr}[\zeta \mid \zeta, \varepsilon](1-\zeta)+\operatorname{Pr}\left[\zeta^{\prime} \mid \zeta, \varepsilon\right]\right] \cdot M^{*}(1, Y)+(1-\zeta) \operatorname{Pr}[\zeta \mid \zeta, \varepsilon] \zeta \cdot 0 \\
& +\zeta\left[\operatorname{Pr}[\zeta \mid \zeta, \varepsilon](1-\zeta)+\operatorname{Pr}\left[\zeta^{\prime} \mid \zeta, \varepsilon\right]\right] \cdot M^{*}(2, \varphi)+\zeta \operatorname{Pr}[\zeta \mid \zeta, \varepsilon] \zeta \cdot \frac{M^{*}(2, \varphi)}{2}
\end{aligned}
$$

while mutants achieve an average reproductive success of

$$
\left[\operatorname{Pr}\left[\zeta \mid \zeta^{\prime}, \varepsilon\right](1-\zeta)+\operatorname{Pr}\left[\zeta^{\prime} \mid \zeta^{\prime}, \varepsilon\right]\right] \cdot M^{*}(1, Y)+\operatorname{Pr}\left[\zeta \mid \zeta^{\prime}, \varepsilon\right] \zeta \cdot 0
$$

A necessary condition for $\zeta$ to be evolutionarily stable against $\zeta^{\prime}=0$ is that, when evaluated at $\varepsilon=0$, the expression in (74) be at least as large as that in (75), i.e.:

$$
(1-\zeta)^{2} \cdot M^{*}(1, Y)+\zeta(1-\zeta / 2) \cdot M^{*}(2, \varphi) \geq[(1-\sigma)(1-\zeta)+\sigma] \cdot M^{*}(1, Y)
$$

Using (73), this reduces to

$$
0 \geq \zeta \sigma \cdot M^{*}(1, Y)
$$


Given that $\zeta \cdot M^{*}(1, Y)>0$, this inequality is false for any $\sigma>0$.

Claim (iii): $\zeta=1$ is evolutionarily stable if $\sigma<\tilde{\sigma}$ but it is not evolutionarily stable if $\sigma>$ $\tilde{\sigma}$. Suppose that $\zeta=1$ is the resident strategy and that a share $\varepsilon>0$ of the population carries the mutant strategy $\zeta^{\prime} \in[0,1)$. The average reproductive success of a resident (who wins with probability $1 / 2$ when matched with another individual playing Compete and with certainty when matched with an individual playing Surrender) is then

$$
\operatorname{Pr}[\zeta \mid \zeta, \varepsilon] \cdot \frac{M^{*}(2, \varphi)}{2}+\operatorname{Pr}\left[\zeta^{\prime} \mid \zeta, \varepsilon\right] \cdot\left[\zeta^{\prime} \cdot \frac{M^{*}(2, \varphi)}{2}+\left(1-\zeta^{\prime}\right) \cdot M^{*}(2, \varphi)\right],
$$

while that of a mutant is

$$
\zeta^{\prime} \cdot\left[\operatorname{Pr}\left[\zeta \mid \zeta^{\prime}, \varepsilon\right]+\operatorname{Pr}\left[\zeta^{\prime} \mid \zeta^{\prime}, \varepsilon\right] \cdot \zeta^{\prime}\right] \cdot \frac{M^{*}(2, \varphi)}{2}+\left(1-\zeta^{\prime}\right) \cdot \operatorname{Pr}\left[\zeta^{\prime} \mid \zeta^{\prime}, \varepsilon\right]\left(1-\zeta^{\prime}\right) \cdot M^{*}(1, Y)
$$

By continuity of these expressions in $\varepsilon$, a necessary condition for $\zeta=1$ to be evolutionarily stable against $\zeta^{\prime} \in[0,1)$ is that, when evaluated at $\varepsilon=0$, the expression in (78) be at least as large as that in (79). This condition writes

$$
\frac{M^{*}(2, \varphi)}{2} \geq \sigma\left[\left(1-\zeta^{\prime}\right) \cdot M^{*}(1, Y)-\zeta^{\prime} \cdot \frac{M^{*}(2, \varphi)}{2}\right]
$$

Since the right-hand side of this inequality attains its maximum for $\zeta^{\prime}=0$, a necessary and sufficient condition for $\zeta=1$ to be evolutionarily stable against any $\zeta^{\prime} \in[0,1)$ is that it be evolutionarily stable against $\zeta^{\prime}=0$. Replacing $\zeta^{\prime}$ by 0 in the preceding inequality, I conclude that a necessary condition for $\zeta=1$ to be evolutionarily stable against any $\zeta^{\prime} \in[0,1)$ is:

$$
\sigma \leq \frac{M^{*}(2, \varphi)}{2 \cdot M^{*}(1, Y)}
$$

By continuity of the expressions in (78) and (79) in $\varepsilon$ (recall that the conditional probabilities are continuous in $\varepsilon$ ), this condition is also sufficient if it holds as a strict inequality.

\subsection{Proof of Proposition 9}

Suppose that $\zeta=1$ is the resident strategy and that a share $\varepsilon>0$ of the population carries the mutant strategy $\zeta^{\prime} \in[0,1)$. The average reproductive success of a resident (who wins with probability $1 / 2$ when matched with another individual playing Compete and with certainty when matched 
with an individual playing Surrender) is then

$$
\begin{aligned}
& \operatorname{Pr}[\zeta \mid \zeta, \varepsilon] \cdot \frac{M^{*}(1, \varphi)+M^{*}(1, \varphi-\Delta)}{2} \\
& +\operatorname{Pr}\left[\zeta^{\prime} \mid \zeta, \varepsilon\right] \cdot\left[\zeta^{\prime} \cdot \frac{M^{*}(1, \varphi)+M^{*}(1, \varphi-\Delta)}{2}+\left(1-\zeta^{\prime}\right) \cdot M^{*}(1, \varphi)\right],
\end{aligned}
$$

while that of a mutant is

$$
\begin{aligned}
& \zeta^{\prime} \cdot\left[\operatorname{Pr}\left[\zeta \mid \zeta^{\prime}, \varepsilon\right]+\operatorname{Pr}\left[\zeta^{\prime} \mid \zeta^{\prime}, \varepsilon\right] \cdot \zeta^{\prime}\right] \cdot \frac{M^{*}(1, \varphi)+M^{*}(1, \varphi-\Delta)}{2} \\
& +\left(1-\zeta^{\prime}\right) \cdot \operatorname{Pr}\left[\zeta^{\prime} \mid \zeta^{\prime}, \varepsilon\right]\left(1-\zeta^{\prime}\right) \cdot M^{*}(1, Y) .
\end{aligned}
$$

By continuity of these expressions in $\varepsilon$, a necessary condition for $\zeta=1$ to be evolutionarily stable against $\zeta^{\prime} \in[0,1)$ is that, when evaluated at $\varepsilon=0$, the expression in (83) be at least as large as that in (84). This condition writes

$$
\frac{M^{*}(1, \varphi)+M^{*}(1, \varphi-\Delta)}{2} \geq \sigma M^{*}(1, Y)-\sigma \zeta^{\prime}\left[\frac{M^{*}(1, \varphi)+M^{*}(1, \varphi-\Delta)}{2}+M^{*}(1, Y)\right]
$$

Since the right-hand side of this inequality attains its maximum for $\zeta^{\prime}=0$, a necessary and sufficient condition for $\zeta=1$ to be evolutionarily stable against any $\zeta^{\prime} \in[0,1)$ is that it be evolutionarily stable against $\zeta^{\prime}=0$. Replacing $\zeta^{\prime}$ by 0 in the preceding inequality, I conclude that a necessary condition for $\zeta=1$ to be evolutionarily stable against any $\zeta^{\prime} \in[0,1)$ is:

$$
\sigma \leq \frac{M^{*}(1, \varphi)+M^{*}(1, \varphi-\Delta)}{2 \cdot M^{*}(1, Y)}
$$

By continuity of the expressions in (78) and (79) in $\varepsilon$ (recall that the conditional probabilities are continuous in $\varepsilon$ ), this condition is also sufficient if it holds as a strict inequality. 


\section{References}

Alesina A., P. Giuliano, and N. Nunn (2013) "On the origins of gender roles: women and the plough," Quarterly Journal of Economics, 128, 469-530.

Alger, I., and D. Cox (2013) "The evolution of altruistic preferences: mothers versus fathers," Review of Economics of the Household, 11, 421-446.

Alger I., P. Hooper, D. Cox, J. Stieglitz, and H.S. Kaplan (2020). "Paternal provisioning results from ecological change," Proceedings of the National Academy of Sciences, 117, 10746-10754.

Alger, I., and J.W. Weibull (2010) "Kinship, incentives and evolution," American Economic Review, $100,1725-1758$.

Alger, I. and J.W. Weibull (2013) "Homo Moralis-preference evolution under incomplete information and assortative matching," Econometrica, 81, 2269-2302.

Alger, I., and J.W. Weibull (2019) "Evolutionary Models of Preference Formation," Annual Review of Economics, 11, 329-354.

Alger, I. and J.W. Weibull, and L. Lehmann (2020) "Evolution of preferences in group-structured populations: genes, guns, and culture," Journal of Economic Theory, 185, 104951.

Alvergne, A., C. Faurie, and M. Raymond (2009) "Variation in testosterone levels and male reproductive effort: Insight from a polygynous human population,"Hormones and Behavior, 56(5), 491-497.

Anderson, K. (2006) "How well does paternity confidence match actual paternity? Evidence from worldwide nonpaternity rates," Current Anthropology, 47, 513 -520.

Apicella, C.L., and A. Dreber (2015) "Sex differences in competitiveness: hunter-gatherer women

and girls compete less in gender-neutral and male-centric tasks," Adaptive Human Behavior and Physiology, 1, 247-269.

Ashraf, Q., and O. Galor (2013) "The 'Out of Africa' hypothesis, human genetic diversity, and comparative economic development" American Economic Review, 103, 1-46.

Balaresque, P., N. Poulet, S. Cussat-Blanc, P. Gerard, L. Quintana-Murci, E. Heyer, and M.A. Jobling (2015) "Y-chromosome descent clusters and male differential reproductive success: young lineage expansions dominate Asian pastoral nomadic populations," European Journal of Human Genetics, 23, 1413-1422.

Barber, N. (2008) "Explaining cross-national differences in polygyny intensity - Resource-defense, sex ratio, and infectious diseases," Cross-Cultural Research, 42, 103-117.

Becker, A., B. Enke, and A. Falk (2020) "Ancient origins of the global variation in economic preferences," AEA Papers and Proceedings, 110, 319-23.

BenYishay, A., P. Grosjean, J. Vecci (2019) "The fish is the friend of matriliny: reef density and matrilineal inheritance," Journal of Development Economics, 127, 234-249.

Bergstrom, T.C. (1994a) "Primogeniture, monogamy and reproductive success in a stratified society," Working paper, University of Michigan.

Bergstrom, T.C. (1994b) “On the economics of polygyny,” Working paper, University of Michigan. Bergstrom, T.C. (1995) "On the evolution of altruistic ethical rules for siblings," American Economic Review 85, 58-81. 
Bergstrom, T.C. (1996) "Economics in a family way," Journal of Economic Literature, 34, 1903-1934. Bergstrom, T.C. (2003) "The algebra of assortative encounters and the evolution of cooperation," International Game Theory Review 5, 211-228.

Bertrand, M. (2011) “New Perspectives on Gender,"in Ashenfelter, O. and D. Card, eds., Handbook of Labor Economics, Amsterdam: North-Holland.

Betzig, L. (1992). "Roman monogamy, " Ethology and Sociobiology, 13(5), 351-383.

Bisin, A., and T. Verdier (2001) "The Economics of Cultural Transmission and the Dynamics of Preferences," Journal of Economic Theory, 97(2), 298-319.

Bowles, S., and J.-K. Choi (2019) "The Neolithic agricultural revolution and the origins of private property, " Journal of Political Economy, 127, 2186-2228.

Cavalli-Sforza, L.L., and W.F. Bodmer (1971) The Genetics of Human Populations. San Francisco: W.H. Freeman.

Clutton-Brock T.H. (1991) The Evolution of Parental Care. Princeton: Princeton University Press.

Croson, R., and U. Gneezy (2009) "Gender Differences in Preferences, " Journal of Economic Literature, 47, 448-74.

Datta Gupta, N., A. Poulsen, and M.C. Villeval (2013) "Gender matching and competitiveness: experimental evidence," Economic Inquiry, 51, 816-E35.

Dekel, E., J.C. Ely, and O. Yilankaya (2007) "Evolution of preferences," Review of Economic Studies, 74, 685-704.

De La Croix, D., and F. Mariani (2015) "From polygyny to serial monogamy: a unified theory of marriage institutions," Review of Economic Studies, 82(2), 565-607.

De Waal, F.B.M., and S. Gavrilets (2013) "Monogamy with a purpose," Proceedings of the National Academy of Sciences, 110(38), 15167-15168.

Durante, R., and J. Buggle (2020) "Climatic risk, cooperation, and the co-evolution of culture and institutions," Economic Journal.

Edlund, L., and H. Ku (2013) "The African slave trade and the curious case of general polygyny," Munich Personal RePEc Archive 52735.

Falk, A., A. Becker, T. Dohmen, B. Enke, D. Huffman, and U. Sunde (2018) "Global evidence on economic preferences," Quarterly Journal of Economics, 133, 1645-1692.

Fisher H. (2016) Anatomy of Love. New York: Norton.

Fisher R.A. (1930) The Genetical Theory of Natural Selection. Oxford: Clarendon Press.

Fortunato, L. (2011) "Reconstructing the history of marriage strategies in Indo-European-speaking societies: monogamy and polygyny," Human Biology, 83, 87-105.

Francesconi, M., C. Ghiglino, and M. Perry (2016) “An evolutionary theory of monogamy," Journal of Economic Theory, 166, 605-628.

Frank, R.H. (1987) "If Homo Economicus could choose his own utility function, would he want one with a conscience?" American Economic Review, 77, 593-604.

Frank, S.A. (1998) Foundations of Social Evolution. Princeton, NJ: Princeton University Press.

Gavrilets, S. (2012) "Human origins and the transition from promiscuity to pair bonding," Proceedings of the National Academy of Sciences, 109, 9923-9928. 
Gettler, L.T., T.W. McDade, A.B. Feranil, and C.W. Kuzawa (2011) "Longitudinal evidence that fatherhood decreases testosterone in human males," Proceedings of the National Academy of Sciences, 108(29), 16194-16199.

Gneezy, U., M. Niederle, and A. Rustichini (2003) "Performance in competitive environments: Gender differences, " Quarterly Journal of Economics, 118, 1049-1074.

Goody, J. (1976) Production and Reproduction. Cambridge: Cambridge University Press.

Gould, E.D., O. Moav, and A. Simhon (2008) "The mystery of monogamy," American Economic Review, 98, 333-57.

Grafen, A. (1979). "The hawk-dove game played between relatives," Animal Behaviour, 27, 905-907. Grosjean, P., and R. Khattar (2019) "It's raining men! Hallelujah? The long-run consequences of male-biased sex ratios," Review of Economic Studies, 86, 723-754.

Güth, W., and M. Yaari (1992) "An evolutionary approach to explain reciprocal behavior in a simple strategic game," in U. Witt (Ed.), Explaining Process and Change - Approaches to Evolutionary Economics. Ann Arbor MI: University of Michigan Press.

Grossbard, A. (1976) "An economic analysis of polygyny: The case of Maiduguri," Current Anthropology 17, 701-707.

Grossbard, A. (1980) “The economics of polygamy," Research in Population Economics 2, 321-350. Hames, R. (1996). "Costs and benefits of monogamy and polygyny for Yanomamö women," Ethology and Sociobiology, 17, 181-199.

Hamilton, W.D. (1964) "The genetical evolution of social behaviour," Journal of Theoretical Biology 7, 1-52.

Hartl, D.L., and A.G. Clark (2007) Principles of Population Genetics (4th edition). Sunderland, MA: Sinauer and Associates.

Heifetz, A., C. Shannon, and Y. Spiegel (2007) "What to maximize if you must," Journal of Economic Theory, 133, 31-57.

Helle, S., J.E. Brommer, J.E. Pettay, V. Lummaa, M. Enbuske, and J. Jokela (2014) "Evolutionary demography of agricultural expansion in preindustrial northern Finland," Proceedings of the Royal Society B: Biological Sciences, 281(1794).

Henrich, J., R. Boyd, and P.J. Richerson (2012) “The puzzle of monogamous marriage," Philosophical Transactions of the Royal SocietyB, 367, 657-669.

Hrdy, S.B. (1999) Mother Nature: Maternal Instincts and how they Shape the Human Species. New York: Ballantine Books.

Jensen, M.K., and A. Rigos (2018) "Evolutionary games and matching rules," International Journal of Game Theory, 47(3), 707-735.

Kaplan, H.S., Hill, K., Hurtado, A. M. \& Lancaster, J. B. (2001) "The embodied capital theory of human evolution," in Ellison, P.T. (Ed.) Reproductive ecology and human evolution (pp. 293 - 317), Hawthorne, NY: Aldine de Gruyter.

Karmin, M., L. Saag, M. Vicente, M.A. Wilson Sayres, M. Järve, U. Gerst Talas, ..., and T. Kivisild (2015) "A recent bottleneck of Y chromosome diversity coincides with a global change in culture," Genome Research, 25, 459-466 
Klug, H. (2018) "Why monogamy? A review of potential ultimate drivers," Frontiers in Ecology and Evolution, 6, Article 30.

Knight, E.L., A. Sarkar, S. Prasad, and P.H. Mehta (2020) "Beyond the challenge hypothesis: The emergence of the dual-hormone hypothesis and recommendations for future research," Hormones and Behavior, 104657.

Koçkesen, L., E.A. Ok, and R. Sethi (2000a) “The Strategic Advantage of Negatively Interdependent Preferences," Journal of Economic Theory, 92, 274-299.

Koçkesen, L., E.A. Ok, and R. Sethi (2000b) "Evolution of Interdependent Preferences in Aggregative Games," Games and Economic Behavior 31, 303-310.

Kramer, K.L., and R.D Greaves (2011) "Postmarital residence and bilateral kin associations among hunter-gatherers: Pumé foragers living in the best of both worlds, " Human Nature, 22(1-2), 41-63. Kramer, K.L., R. Schacht, and A. Bell (2017) "Adult sex ratios and partner scarcity among huntergatherers: implications for dispersal patterns and the evolution of human sociality," Philosophical Transactions of the Royal Society B: Biological Sciences, 372(1729), 20160316.

Lagerlöf, N.-P. (2005) "Sex, equality, and growth," Canadian Journal of Economics, 38, 807-831. Lagerlöf, N.-P. (2010) "Pacifying monogamy," Journal of Economic Growth, 15, 235-262.

Lee, G.R., and L.B. Whitbeck (1990) "Economic systems and rates of polygyny," Journal of Comparative Family Studies, 21, 13-24.

Nitsch, A., V. Lummaa, and C. Faurie (2016) "Sibship effects on dispersal behaviour in a preindustrial human population," Journal of Evolutionary Biology, 29(10), 1986-1998.

Orians G.H (1969) “On the evolution of mating systems in birds and mammals, " American Naturalist, 103, 589 - 603 .

Rayo, L, and G.S. Becker (2007) "Evolutionary efficiency and happiness," Journal of Political Economy, 115, 302-337.

Robson, A.J. (2001) “The biological basis of economic behavior," Journal of Economic Literature, 39, 11-33.

Robson, A.J. (2002) “Evolution and human nature," Journal of Economic Perspectives, 16, 89-106. Robson, A.J., and L. Samuelson (2011) "The evolutionary optimality of decision and experienced utility," Theoretical Economics, 6, 311-339.

Ross, C.T., M. Borgerhoff Mulder, S.-Y. Oh, S. Bowles, B. Beheim, J. Bunce, . . . J. Ziker (2018). "Greater wealth inequality, less polygyny: rethinking the polygyny threshold model," Journal of The Royal Society Interface, 15, 1-15.

Rousset, F. (2004) Genetic Structure and Selection in Subdivided Populations. Princeton, NJ: Princeton University Press.

Seabright, P. (2004) The Company of Strangers: A Natural History of Economic Life. Princeton and Oxford: Princeton University Press.

Simmons, L.W., R.C. Firman, and G. Rhodes (2004) "Human sperm competition: Testis size, sperm production and rates of extrapair copulations," Animal Behaviour, 68, 297-302.

Spolaore, E., and R. Wacziarg (2009) "The Diffusion of Development," Quarterly Journal of Economics, 124, 469-529. 
Tertilt, M. (2005) "Polygyny, fertility, and savings," Journal of Political Economy, 113, 1341-71.

Tertilt, M. (2006) "Polygyny, women's rights, and development," Journal of the European Economic Association, 4, 523-30.

Tinbergen, N. (1963) "On aims and methods of Ethology," Zeitschrift für Tierpsychologie, 20, 410433.

Van Schaik, C.P., and C.H. Janson (2000) Infanticide by Males and its Implications. Cambridge: Cambridge University Press.

Van Schaik, C.P. (2016) The Primate Origin of Human Behavior. Hoboken, NJ: Wiley-Blackwell.

Weibull, J.W. (1995) Evolutionary Game Theory. Cambridge MA: MIT Press.

Wright, S. (1931): "Evolution in Mendelian populations," Genetics 16, 97-159.

Wu, J. (2020): "Labelling, homophily and preference evolution," International Journal of Game Theory 49, 1-22.

Zeng, T.C., Aw, A.J. \& Feldman, M.W. (2018) "Cultural hitchhiking and competition between patrilineal kin groups explain the post-Neolithic Y-chromosome bottleneck," Nature Communications, 9, 2077. 